\title{
Tempel des Ernstes und des fake. Der Raffael-Saal in der Orangerie zu Potsdam, ein Kopienmuseum im Zeitalter der technischen Reproduzierbarkeit
}

\section{Book Part, Published Version}

This version is available at http://dx.doi.org/10.14279/depositonce-5604.

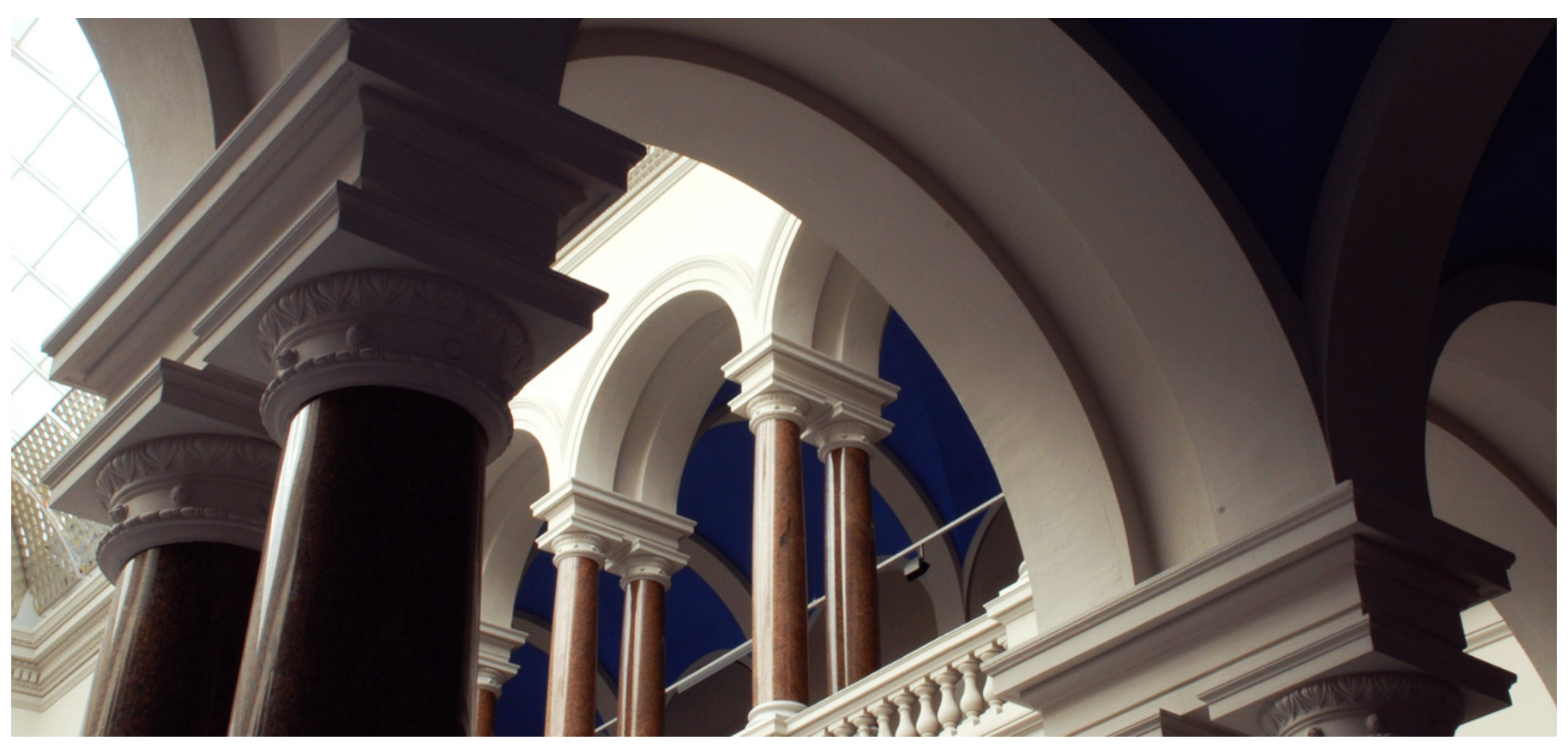

\section{Suggested Citation}

Savoy, Bénédicte: Tempel des Ernstes und des fake. Der Raffael-Saal in der Orangerie zu Potsdam, ein Kopienmuseum im Zeitalter der technischen Reproduzierbarkeit. - In: Heß, Gilbert; Agazzi, Elena;

Décultot, Élisabeth (eds.): Raffael als Paradigma : Rezeption, Imagination und Kult im 19. Jahrhundert . Berlin/Boston : De Gruyter, 2012.- ISBN: 978-3-11-025563-8. - pp. 201-236. - DOI:

https://doi.org/10.1515/9783110255638.201. 


\section{Tempel des Ernstes und des fake Der Raffael-Saal in der Orangerie zu Potsdam, ein Kopienmuseum im Zeitalter der technischen Reproduzierbarkeit}

Ein Zeitgenosse nannte ihn Anfang der 1860er Jahre ein „epochemachendes Ereignis “. ${ }^{1}$ Heute gehört er zu den kaum beachteten Museumsinszenierungen des 19. Jahrhunderts in Deutschland: Der seit Herbst 1858 öffentlich zugängliche Raffael-Saal in der Neuen Orangerie zu Potsdam ist freilich ein kurioser Ort, ein Tempel des Ernstes und ein Tempel des fake zugleich, in dem sich der Raffaelkult des preußischen Königshauses, ja des gesamten deutschen 19. Jahrhunderts verfestigt hat: Beste Oberlichtbeleuchtung, rote Wandbespannung, Stuckdecke, Marmorboden, reicher Skulpturenschmuck, ein einladendes Sofa in der Mitte und an die fünfzig Gemälde in goldenen Rahmen an den Wänden.

Wären diese Gemälde nicht merkwürdig geschliffene Kopien nach Raffael in irritierenden Proportionen und wüßte man nicht, daß rechts und links des bekuppelten Saals die exotischen Kübelpflanzen des Parks von Sanssouci in langen weiten Hallen ihre Winterquartiere haben, so könnte man sich im Dresdner Semperbau wähnen, in einem Saal des Kunsthistorischen Museums in Wien oder gar in Klenzes Petersburger Neuer Eremitage. Der Raffael-Saal zu Potsdam ist ein Museumssaal des 19. Jahrhunderts wie er im Buche steht, er erzählt die eigenartige Ge-

${ }^{1}$ Bussler, Robert: Der Rafael-Saal. Verzeichnis der im königlichen Orangeriehause zu Sans-Souci auf allerböchsten Befebl aufgestellten Copien nach Gemälden von Rafael Sanzio. Nachdr. der 2. Auflage [1861]. Bildanhang und Kommentar von Gerd Bartoschek. Potsdam 1983, S. 62. Zum Raffaelsaal, vgl. Windholz, Angela: „Raffael als alter ego der Preußischen Monarchie. Zur bildpolitischen Intention des Raffaelsaals in Sanssouci“, in: Marburger Jabrbuch für Kunstwissenschaft, Bd. 35 (2008), S. 215-265. Des Weiteren Bartoschek, Gerd / Hüneke, Saskia / Paepke, Karola: „Ein Zeugnis schwärmerischer Verehrung. Geschichte und Restaurierung des Raffaelsaales", in: MuseumsJournal, 7/1993, 2, S. 42-44. 


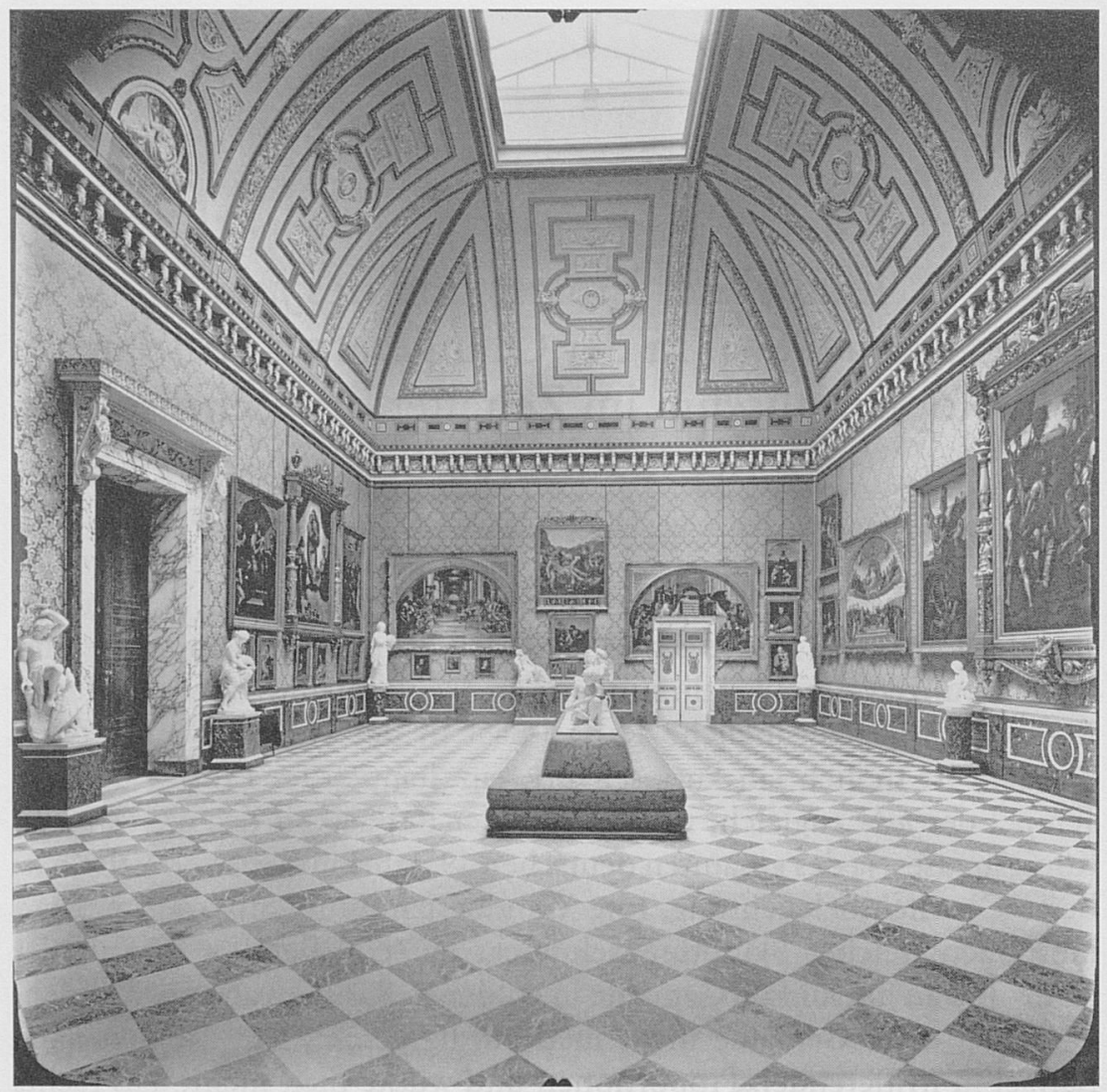

Abb. 1: Potsdam, Sanssouci, Neue Orangerie, Raffelsaal

(Foto Preußische Meßbildanstalt, 1. Drittel 20. Jh.).

schichte einer ausgestorbenen Museumsform, die des Kopienmuseums. ${ }^{2}$ Er steht allerdings einsam da - scheint weder funktional noch formal zum Schloßkomplex der Orangerie zu gehören. Ideell schon: Er bildet das Herz der von Friedrich August Stüler, Peter Joseph Lenné und Friedrich Wilhelm IV. um 1850 konzipierten Architektur und Gartenlandschaft am nördlichen Rand des Parks von Sanssouci - ein kraftvolles Bekenntnis des preußischen Königshauses zur Kunst und Architektur der

2 Vgl. zuletzt Dlugaiczyk, Martina / Markschies, Alexander: Mustergültig. Gemäldekopien in neuem Licht. Das Reiff-Museum der RWTH Aachen. Katalog zur Ausstellung im Suermondt-Ludwig-Museum, Aachen 2008/09. München 2008. 


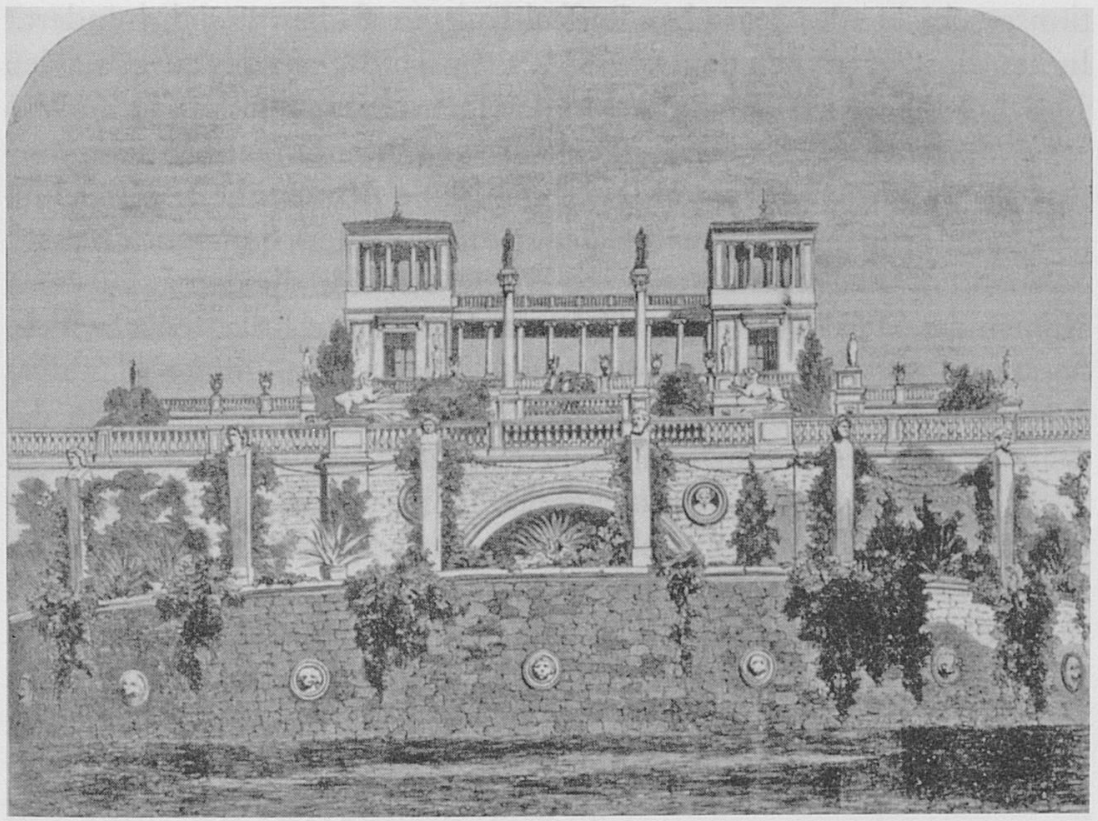

Abb. 2: Potsdam, Sanssouci, Die Neue Orangerie mit Terrassen.

Holzstich nach einer Fotografie von H. Selle, 1865

italienischen Renaissance, deren unbezweifelter Gipfel im 19. Jahrhundert Raffael war. ${ }^{3}$ Er ist ein Museum gewordener, explizit als solcher deklarierter Versuch, gegen den durch die Verbreitung der Fotografie verursachten Verlust der Aura von Kunstwerken zu wirken.

\section{Neuschöpfung Raffaels}

„Eine entscheidende Voraussetzung für die Entstehung des deutschen Raffael-Kults bildet die geringe Repräsentanz von Raffaels Werk in deutschen Museen“, schreibt Ernst Osterkamp in seiner Studie zur Funk-

${ }^{3}$ Zur Begründung der Raffaelbegeisterung aus dem Geiste der deutschen Romantik, vgl. vor allem Osterkamp, Ernst: „Raffael-Forschung von Fiorillo bis Passavant", in: Studi germanici, nuova serie, XXXVIII/2000, 3, S. 403-426; ferner Schröter, Elisabeth: „Raffael-Kult und Raffael-Forschung. Johann David Passavant und seine Raffael-Monographie im Kontext der Kunst und Kunstgeschichte seiner Zeit“, in: Römisches Jabrbuch, 26/1990, S. 303-393. 
tionsgeschichte des deutschen Raffael-Kults im frühen 19. Jahrhundert. ${ }^{4}$ In der sonst so reichen deutschen Museumslandschaft des ausgehenden 18. Jahrhunderts glänzte Raffael in der Tat - abgesehen von der Sixtinischen Madonna in Dresden, die gerade wegen ihrer Singularität zum heiBen Kultobjekt erhoben wurde - durch seine Abwesenheit. ${ }^{5}$ Zwar hingen so gut wie überall, in den Galerien zu München, Sanssouci, Kassel, Salzdahlum bei Braunschweig und Wien, Gemälde die den auratischen Namen des Malerstars trugen. Daß es sich dabei um Etikettenschwindel handelte, war den Museumsbesuchern aber schon immer klar. Das brachte der Berliner Verleger Friedrich Nicolai bereits zu Beginn der 1780 er Jahre auf den Punkt, als er die noch nicht eröffnete Hofgartengalerie in München besuchte und schrieb: „Eine Maria mit dem toten Jesus im Arm soll von Raphael sein. Ich bin kein großer Kenner, doch nach dem zu urteilen, was ich von Raphael in Dresden und Wien gesehen habe, bezweifle ich, daß das Münchner Bild von ihm stammt. Da aber anscheinend jede Galerie ihren Raphael haben muß, so will ich nicht weiter widersprechen" ${ }^{\text {" }}$

Jeder Galerie ihren Raffael! Es wäre natürlich bequem, die weitgehende Abwesenheit von Raffaels Werk in deutschen Sammlungen auch als direkte Voraussetzung für die Entstehung des Potsdamer Kopienkuriosums verantwortlich zu machen. Vieles spricht dafür: In Ermangelung von Originalen, angesichts leerer Staatskassen und des engen Angebots auf dem Kunstmarkt um 1800 entsteht in der preußischen Hauptstadt eine Kopiensammlung als Substitut, ein Raffaeltempel mit Placeboeffekt. In Berlin hing in der Tat lange kein Originalgemälde von Raffael der Ankauf der fünf Madonnenbilder für die königliche Sammlung, die heute noch die einzigen Raffaels in Berlin sind, gelang bekanntlich erst zwischen 1821 und 1854.7 Trotzdem darf man die Raffaelkopien des Raf-

${ }^{4}$ Osterkamp: „Raffael-Forschung“, S. 404; s. ferner Osterkamp, Ernst: „Maria, Auguste und die Madonna. Die Bedeutung Raffaels für Goethe, erläutert am Beispiel von zwei Gedichten an Personen", in: Gerhard Neuman / David E. Wellbery (Hrsg.): Die Gabe des Gedichts. Goethes Lyrik im Wechsel der Töne. Freiburg 2008, S. $185-207$.

5 Savoy, Bénédicte (Hrsg.): Tempel der Kunst. Die Entstehung des öffentlichen Museums in Deutschland. 1701-1815. Mainz 2006, passim.

${ }^{6}$ Nicolai, Friedrich: Beschreibung einer Reise durch Deutschland und die Schweiz im Jahr 1781. Berlin/Stettin 1784, S. 47 f., abgedruckt in: Savoy (Hrsg.): Tempel der Kunst, S. 529 .

7 Vgl. Stockhausen, Tillmann von: Gemäldegalerie Berlin. Die Geschichte ibrer Erwerbungspolitik. Berlin 2000, S. 87. 
faelsaals nicht primär als einen Ersatz für fehlende Originale betrachten. Es ist nämlich zu einfach, immer wieder auf die generelle Akzeptanz von Kopien in vorhergehenden Jahrhunderten hinzuweisen, um die Entstehung von Kopiensammlungen allgemein, und die des Raffaelsaals im Besonderen, retrospektiv zu erklären. Um 1800 wußte das aufgeklärte Publikum sehr wohl zwischen dem Wert einer Kopie und dem Wert eines Originals zu unterscheiden. Ja schon um 1700 häuften sich die Bemerkungen von Galeriebesuchern, die Kopien als minderwertig abstempelten: Ein schönes Beispiel dafür ist die Bemerkung des Sammlers und Juristen Zacharias Conrad von Uffenbach, der im Jahre 1709 vergleichend über die Galerien in Düsseldorf und Salzdahlum bei Braunschweig schrieb: „Es ist zwar ein schöner Vorrath von Gemählden allhier doch ist in Salzthalen [sic] ein weit mehrers. Herr Friderici wollte zwar behaupten, daß daselbst gar viele Copien, da hingegen hier lauter Originalien wären, allein ich hielte es vor Geschwätze. "8 Kurzum: Die Akzeptanz von Kopien als Ersatz für Originale war um 1800 wahrscheinlich nicht viel höher als heute. Wer als König im großen Stil Kopien bestellte und sammelte, tat es nicht mit der primitiven Absicht, Originale zu ersetzen. Die Raffaelkopien im Potsdamer Orangerieschloß erzählen mehr als die Geschichte eines gefüllten Vakuums. Sie sind das Zeugnis einer ganz besonderen Form der Aneignung, die sich weder literarisch noch kunstwissenschaftlich sondern handwerklich und piktural, Millimeter für Millimeter, gestaltete.

So betrachtet, ist es kein Wunder, wenn unter den 50 Kopien im Potsdamer Raffaelsaal kaum eine „alte“ Kopie hängt - genau genommen ist es nur eine einzige: die Reduktion auf Leinwand des Attilafresko aus der Stanza d'Eliodoro im Vatikan (Kopie aus dem 17. Jahrhundert). ${ }^{9}$ Alle anderen „Raffaels“ dort sind zwischen 1801/1804 und 1861 entstanden. Ob es in den ersten Jahrzehnten des 19. Jahrhunderts für das preußische Königshaus eine Option gewesen wäre, den europäischen Kunstmarkt nach guten historischen, alt anmutenden Raffaelkopien durchforsten zu lassen, darüber läßt sich nur spekulieren. Im Kontext ihrer Überlegungen zur Eröffnung des königlichen Museums am Lustgarten plädierten Karl Friedrich Schinkel und Gustav Friedrich Waagen jedenfalls 1828 dafür, alte „Replichen“ zu erwerben, „die in einem hohen Grade den Geist des

${ }^{8}$ Uffenbach, Zacharias Conrad von: Merkwürdige Reisen durch Niedersachsen Holland und Engelland, 1709. 3 Bde. Ulm 1753/54. Bd. 1, S. 725-743, abgedruckt in: Savoy (Hrsg.): Tempel der Kunst, S. 384.

9 Bussler: Der Rafael-Saal, 1861, Anhang (Bartoschek), S. XIII, Nr. 37/20. 
Originals zu athmen und auch technisch in ähnlicher Art behandelt zu sein [pflegen], so daß sie bei der seltenen Gelegenheit der Originale selbst habhaft zu werden, immer sehr willkommen sein müssen. "10 Das war eine Richtlinie für das Museum. Die Potsdamer Raffaelsammlung aber - oder genauer gesagt: die Raffaelkopisten und die Raffaelsammler, die zu dieser sonderbaren Sammlung beitrugen, wollten keinen dreihundert Jahre alten Raffael, der nach altem Geist riecht. Sie wollte ihn jung, frisch, neugeboren und leuchtend. Mehr noch: Sie wollten ihn selber erzeugen - ja er selber sein.

Von dieser Logik zeugen viele konvergierende Quellen. Aufschlußreich ist zum Beispiel eine der seltenen Selbstauskünfte einer professionellen Kopistin, der Dresdnerin Therese aus dem Winckel, die im Jahre 1806 in langen Briefen aus Paris ihr Talent und den Sinn ihrer Arbeit anpries. Sie verglich das Kopieren mit einer Verjüngungskur für Gemälde, einer Möglichkeit „die ewig schönen Urbilder [...] verjüngt und sie schöner als kalte Schattenbilder es vermögen, spätern Jahrhunderten zu überliefern". ${ }^{11}$ Raffael wurde bunt, das Kopieren in Öl und im Originalformat trat in Konkurrenz zu den traditionellen, farblosen und reduzierenden Reproduktionsmedien („kalte Schattenbilder“). Es ist kein Zufall, wenn der Kopienboom der Jahre um 1800 mit der zum selben Zeitpunkt hitzig geführten Diskussion um die Zweckmäßigkeit von Restaurierungen einherging. ${ }^{12}$ Sowohl beim Kopieren in Öl als auch beim Restaurieren sollte das Historische und Zeitliche der Kunstwerke möglichst unsichtbar, ja rückgängig gemacht werden (die immer wiederkehrende Metaphorik der „Verjüngung" mit ihren sakral anmutenden Entsprechungen „Wiederauferstehung“, „neues Leben“ etc. zeugen davon). Dieser Zusammenhang zwischen Kopieraktivität und Restaurierungsproblematik wird übrigens besonders deutlich, wenn man sich die Verwaltungsakten anschaut, welche die Ankunft und Begutachtung der heute im Raffaelsaal

10 Schinkel, Karl Friedrich / Waagen, Gustav Friedrich: „Über die Aufgabe der Berliner Galerie“, August 1828, abgedruckt in: Friedrich Stock: „Urkunden zur Vorgeschichte des Berliner Museums“, in: $J b$. der preußischen Kunstsammlungen 49/1928, Beiheft, S. 65-174 und 51/1930, S. 205-222, hier: S. 222 ff. Vgl. Strittmatter, Anette: Das, Gemäldekopieren' in der deutschen Malerei zwischen 1780 und 1860. Münster 1998, S. 235 f.

11 Briefe der Therese aus dem Winckel an Karl August Böttiger, Paris, 29. Januar 1808. Abgedruckt in: Strittmatter: Das, Gemäldekopieren', S. 304 f., hier: S. 304.

12 Vgl. Wagner, Cornelia: Arbeitsweisen und Anschauungen in der Gemälderestaurierung um 1800 (=Veröffentlichungen des Instituts für Kunsttechnik und Konservierung im Germanischen Nationalmuseum, Bd. 2). München 1988. 
versammelten Kopien dokumentieren. ${ }^{13}$ Traf zum Beispiel in Berlin eine Kopie ein, die als zu dunkel empfunden wurde, so verurteilte man sie a priori als mißlungen. Vor dem geistigen Auge der Deutschen im frühen 19. Jahrhundert, die ja nur in seltenen Fällen authentische Bilder von Raffael gesehen hatten (und dabei übrigens nicht selten enttäuscht gewesen waren), war der „Heiland der Farben" hell - und er hatte auch hell zu sein. Es ist bezeichnend, daß ein Experte wie Aloys Hirt, als er im Herbst 1821 vom preußischen Königshaus gebeten wurde, eine als mißlungen empfundene Kopie des Erzengels Michael durch den jungen Maler Wilhelm Ternite zu begutachten, den Kopisten in Schutz nahm mit dem Argument die Kopie sei treu, das Original aber schlecht erhalten:

[ich] habe die Ehre in Beziehung auf die Copie des Erzengels Michael nach Raphael durch Herrn Ternite gehorsamst zu antworten, daß ich das Originale dieses Gemäldes nie sah, aber aus der Copie schließen muß, daß dasselbe in manchen Stellen sehr nachgedunkelt ist und an der Lebhaftigkeit und Klarheit der Farben viel verloren hat. [...] Die matten Farben [...] müssen auf Rechnung des Originals kommen. ${ }^{14}$

Die Kopie entsprach, so Hirt, dem damaligen Zustand des Werks. Und gerade deswegen fand man sie ja auch schlecht. Von ihr hätte man erwarten können - und das erwartete man auch - daß sie die Verdunkelung rückgängig gemacht hätte. Genauso wie man auch erwartete, daß Hinzufügungen aus späteren Zeiten getilgt, ${ }^{15}$ Rahmen zwar im Stile der Renaissance aber als deutlich erkennbare Neuschöpfungen, die man sogar zu Weltausstellung nach London schicken könnte, ${ }^{16}$ für die Kopien entworfen würden.

${ }^{13}$ Vgl. u.a. Berlin, Geheimes Staatsarchiv, 1. HA Rep. 89 Geh. Zivilkabinett, jüngere Periode, Akten betreffend verschiedene Maler, die u.a. als Kopisten für den Raffael-Saal tätig waren.

14 Gutachten von Aloys Hirt vom 30. September 1821, Berlin, Geheimes Staatsarchiv, 1. HA Rep. 89 Geh. Zivilkabinett, jüngere Periode, Nr. 19810, „Acta des königl. Civil-Cabinets betr. die dem Maler Ternite bewilligten Unterstützungen, die von demselben eingereichen Gemälde und Kunstsachen und sonstige Angelegenheiten derselben 1812-1855", fol. 79. Zu Ternite, der unter anderem als Agent für die Rückforderung von 1806 nach Paris entführten Kunstwerke agierte, vgl. Savoy, Bénédicte: Patrimoine annexé. 2 Bde. Bd. 1, Paris 2003, S. 164.

15 „Der auf dem Vordergrund dargestellte Tisch und die darauf liegende Rose, die dem Bild seinen Namen gegeben hat, sind als Hinzufügungen aus späterer Zeit bei der Kopie weggelassen" bemerkt Gerd Bartoschek in: Bussler: Der Rafael-Saal, 1861, S. XIX, über die reduzierte Kopie der Madonna della Rosa (Raffaelschule, um 1518-1520) von unbekannter Hand.

${ }^{16}$ Laut Gerd Barthoschek (in: Bussler: Der Rafael-Saal, wie Anm. 1), S. XV, wurde z.B. der von Jakob Alberty 1850 nach einem Entwurf von Friedrich August Schü- 


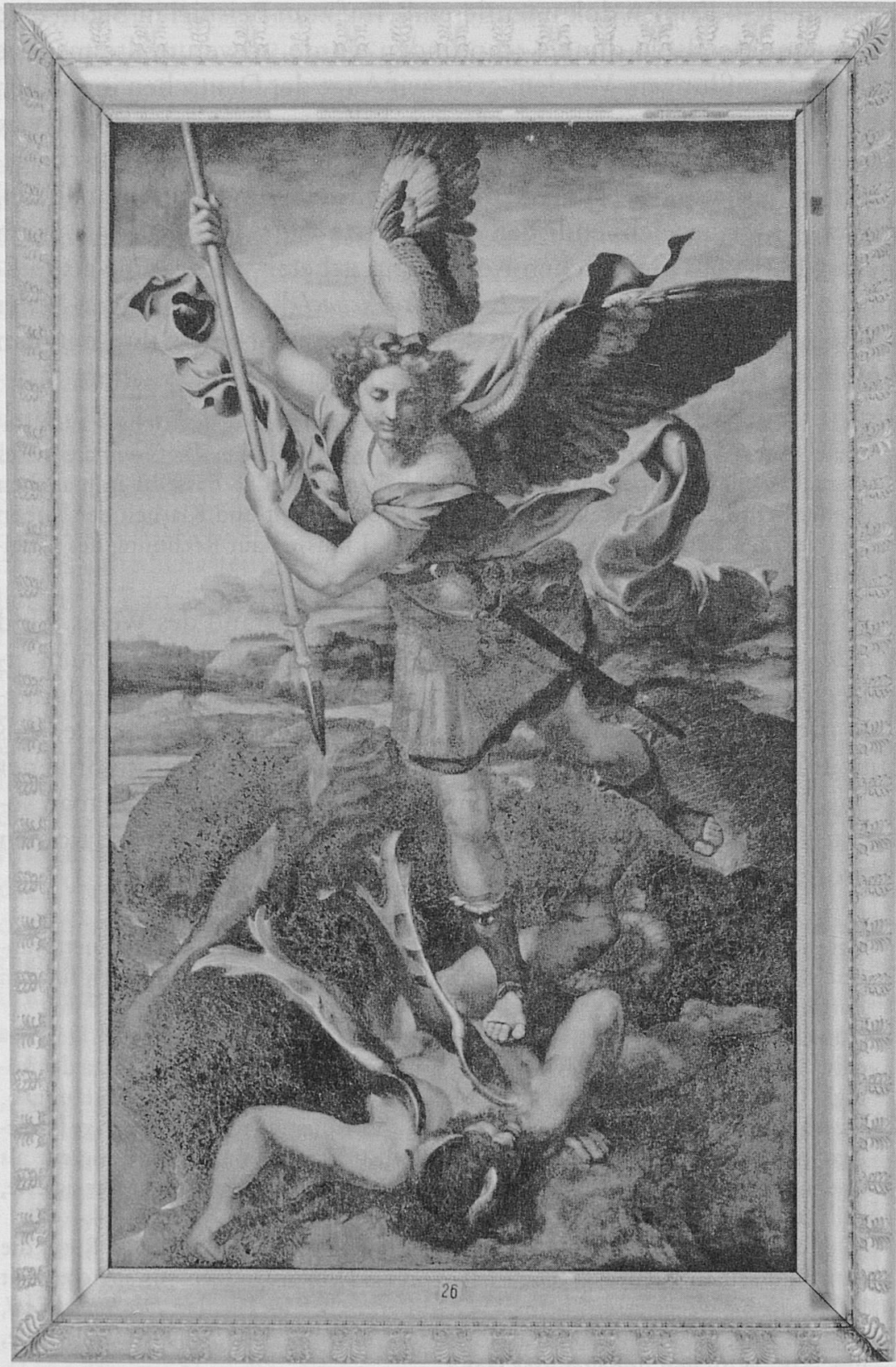

Abb. 3: Wilhelm Ternite: Kopie nach Raffael, Der Erzengel Michael (1518). Paris, Louvre, $1818-1821$ 
Es mag paradox klingen: Die Raffaelkopien, die in diesen ersten Jahrzehnten des 19. Jahrhunderts entstanden und heute im Raffael-Saal zu sehen sind, wollten also im Grunde keine treuen Nachbildungen der in Paris, Rom etc. vorhandenen Originale sein, wie sie um $1800 \mathrm{zu}$ sehen waren, sondern Kopien eines abstrakten, idealisierten, literarisch geprägten Originalzustand des Werks von Raffael - zugespitzt gesagt: Kopien nach mentalen Bildern oder, um mit Ernst Osterkamp zu sprechen, Bilder "eines gereinigten und geläuterten Raffaelbildes", ${ }_{17}$ so etwas also wie das medienkonforme Abbild der zeitgenössischen Raffaelwahrnehmung. In einem kurzen, methodisch erregenden Aufsatz hat Franz Kugler 1851 dieses Phänomen wunderbar erkannt. ${ }^{18}$ Er berichtet darin über eine im Frühjahr 1848 unter vielen anderen Raffaelkopien in Berlin ausgestellte Wiedergabe der Kreuztragung von Raffael (Lo Spasimo, heute Madrid) durch den Berliner Maler und angesehenen Restaurator Jakob Schlesinger (1792-1855): ${ }^{19}$

Unter den Kopien der Staffeleigemälde befand sich auch die Kreuztragung (Spasimo di Sicilia), die in jüngster Zeit durch den Professor Schlesinger, Restaurateur der Gemäldegallerie des Museums, im Auftrage des Königs nach dem in Madrid befindlichen Originale angefertigt ist. [...] Man fand aber nicht, was man erwartet hatte, und ein ziemlich allgemeines Missbehagen war unverkennbar. Viele wussten gar nicht, was sie aus einem Bilde machen sollten, das so auffallend von der raphaelischen Behandlungsweise abwich. Einige trösteten sich kurzweg und meinten, es sei eben eine missrathene Kopie; Andere deuteten, nicht ganz ohne sarkastische Bermerkungen, darauf hin, daß der berühmte Restaurator wohl die

ler entworfene Rahmen für die von Jakob Schlesinger ausgeführte Kopie nach Raffaels Lo Spasimo (um 1516, Madrid, Prado) zur 1851 in London stattfindenden Weltausstellung geschickt. Vgl. auch Roenne, Bettina von: Ein Architekt rabmt Bilder. Karl Friedrich Schinkel und die Berliner Gemäldegalerie. Berlin 2007. Kat. Nr. 65. S. 112-113: Tabernakelrahmen zur Kopie Wilhelm Hensels nach Raffaels „Madonna Conestabile“.

17 Osterkamp: Raffael-Forschung, S. 406.

18 Kugler, Franz: „Einige Bedenken über Raphaels Kreuztragung, nach Maassgabe der Schlesinger'schen Kopie“, in: Deutsches Kunstblatt, 1850, Nr. 14, abgedruckt in: Kugler, Franz: Kleine Schriften und Studien zur Kunstgeschichte. 2. Teil, Stuttgart 1854, S. 594-599. Methodisch erregend, weil Kugler darin versucht, anhand der bloßen Kopie (!) eine Zuschreibung des Gemäldes zu treffen.

19 Schlesinger, Jakob: Kopie nach Raffael, Lo Spasimo (um 1516, Madrid, Prado). Um 1848, Originalgröße, Potsdam, OrangerieSchloß, Raffaelsaal. Vgl. Waagen, Gustav Friedrich: Verzeichniß der Copien und der Kupferstiche nach Raphael so wie einiger Originalzeichnungen dieses Meisters welche in der Rotunde des Königlichen Museum ausgestellt worden sind. Berlin 1848; des Weiteren Bartoschek, Gerd in: Bussler: Der Rafael-Saal, 1861, S. IV-VIII, hier: S. VI. 


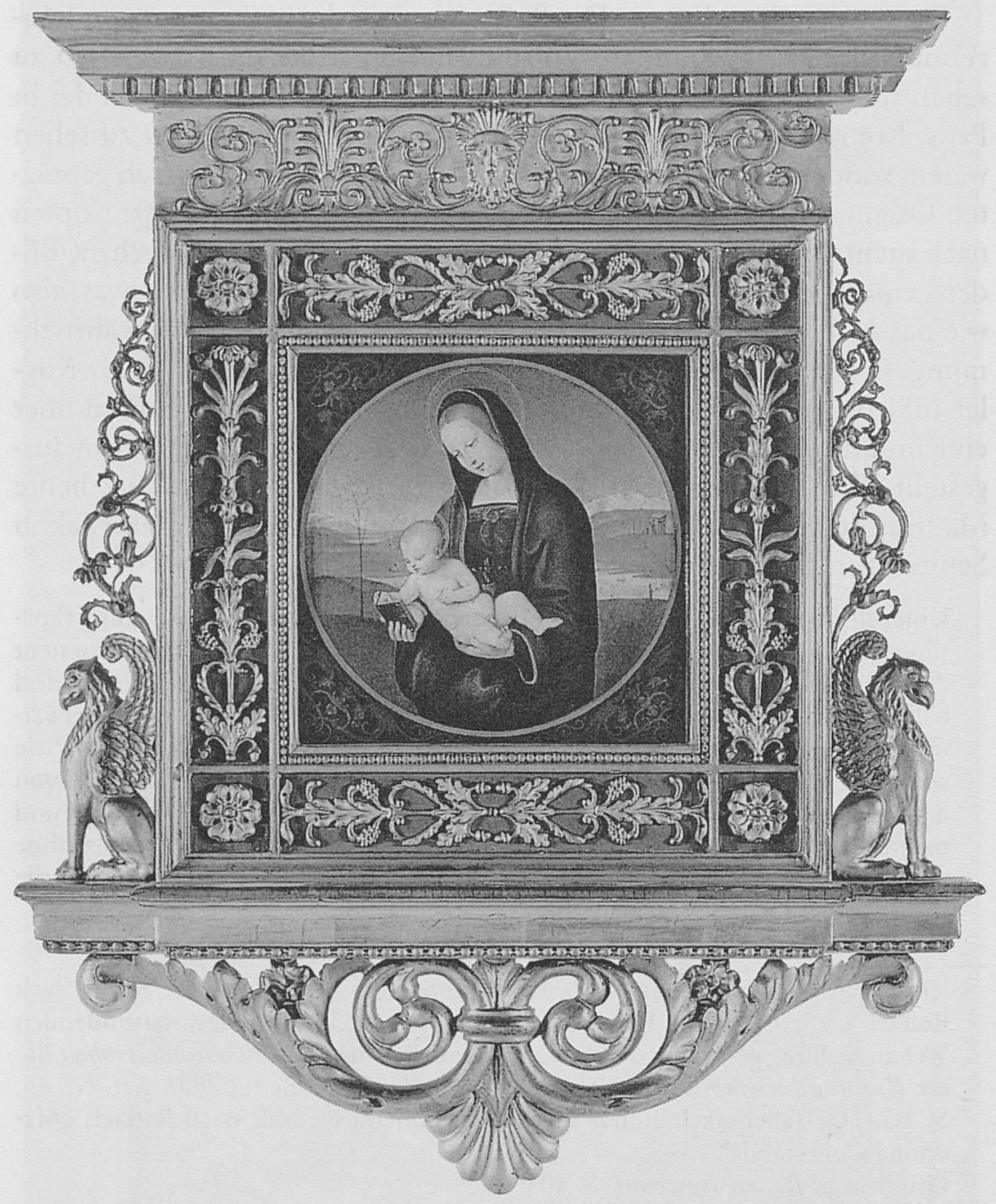

Abb. 4: Karl Friedrich Schinkel: Tabernakelrabmen zur Kopie von Raffaels „Madonna Conestabile"(1504).

Absicht gehabt habe, uns einmal auf eclatante Weise zu zeigen, wie Raphaels Bilder, ehe Zeit und Unverstand sie in ihre dermaligen Zustände versetzt, ursprünglich beschaffen gewesen seien, oder vielleicht gar: wie Raphael eigentlich hätte malen sollen. ${ }^{20}$

${ }^{20}$ Kugler: Einige Bedenken, S. 595. 


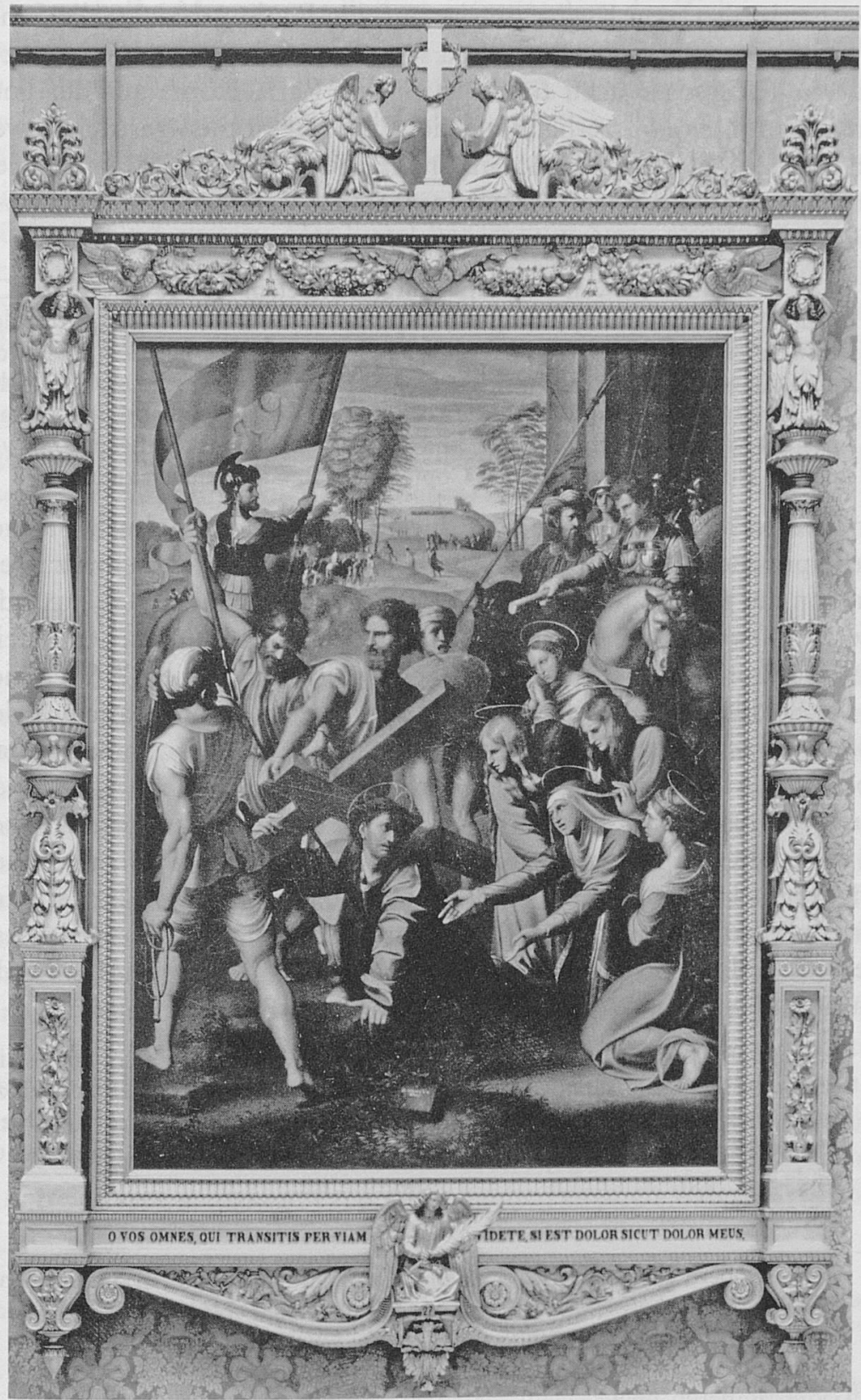

Abb. 5: Jakob Schlesinger: Kopie nach Raffael, Lo Spasimo

(um 1516. Madrid. Prado), um 1848 
Hier ist sie also, von Kugler präzise auf den Punkt gebracht: die Sehnsucht des Kopisten selber Raffael zu sein. Eine Sehnsucht, die in einem anderen Falle sogar dazu führte, daß eine Raffaelkopie aus der Potsdamer Sammlung kurzerhand aus der Liste der Kopien gestrichen werden mußte, weil der Kopist sie übermalt und mit seinem Namen signiert hatte - davon berichtet der Historiograph des Raffaelsaals Robert Bussler im Jahre 1866:

Eine [...] 1814 in Paris von Ternite mit ausgezeichnetem Erfolge ausgeführte Copie der Mittelfigur der Kreuztragung ist leider durch die Schuld des Künstlers selbst dieser Sammlung verloren gegangen, indem derselbe bei Gelegenheit der Ausführung einer Restauration sich veranlaßt fand, sein Bild ganz zu übermalen und dasselbe dadurch so vollständig zu verändern, daß er selbst seinen Namen anstatt des des Rafael daruntersetzte und Se. Majestät den Hochseligen König veranlasste, sein Bild aus der Liste der Copien nach Rafael streichen zu lassen. ${ }^{21}$

Eine Bemerkung am Rande: Die hier diagnostizierte Sehnsucht nach partizipierender Neuschöpfung durch Kopien ist nicht getrennt zu denken von der gerade zu Beginn des 19. Jahrhunderts blühenden Praxis der tableaux vivants, der körperlichen Nachstellung und physischen Aneignung berühmter Kunstwerke durch Schauspieler und Laien.22 Auch hier, an der Schnittstelle zwischen Malerei und Theater, spielte Raffael in den ersten Jahrzehnten des 19. Jahrhunderts, gerade in Berlin, eine zentrale Rolle. Auch hier ging es ausdrücklich um seine physische Verlebendigung, es ging darum, sein Werk für dreißig Sekunden zum neuen Leben zu erwecken, ein Prozeß, der auch mit einem seltsamen Drang einherging, Raffael zu verbessern. Ein Beispiel unter vielen: im März 1812 ließ der Architekt Carl Ferdinand Langhans acht Gemälde, davon vier Raffaels im Berliner Schauspielhaus nachstellen. Zum Heiligen Michael (damals und heute: Louvre) schrieb ein anonymer Berichterstatter:

Die Demoiselle Engel, unsre erste Solotänzerin, gab dieses Bild so, wie es sich von ihr erwarten ließ, sehr befriedigend. Ihr ganzer Wuchs sagte diesem Bilde zu, ihre Haltung war der Idee des Künstlers entsprechend, und eine Ruhe herrschte in dieser schwierigen Stellung, als wenn das Bild lebendig auf der Leinwand dastände. ${ }^{23}$

${ }^{21}$ Bussler, Robert: „Der Rafaelsaal im neuen Orangerie-Gebäude“, in: Louis Schneider (Hrsg.): Mittheilungen des Vereins für die Geschichte Potsdams. Bd. 2, Potsdam 1866, S. 17-22, hier: S. 21.

22 Vgl. Jooss, Birgit: Lebende Bilder. Körperliche Nachabmung von Gruppenbildern in der Goethezeit. Berlin 1999.

${ }^{23}$ W., G.: „Lebende Bilder“, in: Journal für Kunst und Kunstsachen, Künsteleien und Mode, 12/1811, S. 289-309, hier: S. 295. 
Für eine halbe Minute war Raffael auferstanden, das Ziel war erreicht. Bezeichnenderweise gaben solche performances allerdings auch Anlaß zu Kritiken - nicht nur der Darsteller, nein, auch der Gemälde selbst. Im Zuge der Verlebendigung des Heiligen Michael stellte der eben erwähnte Berichterstatter bezeichnenderweise fest, „dass die Rüstung, womit der Engel angethan war, unserm Rafael fast zum Vorwurfe gereichen möchte“. Und weiter: „Der Engel bedarf zum Siege nur innerer Kraft, welche von Gott gegeben, nicht der menschlichen Rüstung. Es drängte sich ferner dem Sinne mächtig auf, wie weit vortheilhafter und poetischer eine Drappirung dem Bilde gewesen sein würde". ${ }^{24}$ Körperliche Aneignung und korrigierender Impetus: sowohl bei der Nachstellung lebender Bilder als auch bei der Herstellung und dem Sammeln unzähliger moderner Kopien nach Raffael ging es um 1800 darum, die historische Distanz zum Idol zu verringern, ja aufzuheben.

\section{Ein kollektives Projekt}

In der spärlich gesäten Literatur zum Raffaelsaal wird in der Regel betont, wie sehr die Potsdamer Kopiensammlung auf die Initiative eines einzelnen Mannes und seiner Eltern zurückgeht, auf die geerbte Raffaelbegeisterung und die religiösen Interessen Friedrich Wilhelms IV von Preußen. ${ }^{25}$ Dies ist natürlich nicht von der Hand zu weisen. Diese biographische, preußenzentrierte Fixierung darf allerdings nicht den Blick für die Tatsache versperren, daß die Potsdamer Raffaelsammlung Teil eines viel breiteren, man möchte sagen: kollektiven Aneignungsprojekts des Werks von Raffael mittels Kopien gewesen ist, das Projekt einer ganzen Generation. Wurde nämlich die älteste „moderne“ Kopie des Raffaelsaals, die Sixtinische Madonna, bekanntlich von der Königin Luise beim prominenten Dresdner Maler und Goetheportraitisten Friedrich Bury im Jahre 1801 in Auftrag gegeben (als Geschenk für ihren Gatten), ${ }^{26}$ so gestalte sich bereits die nächste Erwerbung ganz anders, nämlich auf dem freien Kunstmarkt in Paris. Dort kaufte Friedrich Wilhelm III. 1814

24 Ebd.

25 Vgl. z.B. Bartoschek, Gerd / Hüneke, Saskia / Paepke, Karola: „Der Raffaelsaal: Ein Zeugnis schwärmerischer Verehrung“, Museumsjournal, Heft Nr. II/1993, S. $42-44$.

26 Vgl. Putscher, Marielene: Raphaels Sixtinische Madonna: das Werk und seine Wirkung. Tübingen 1955, S. 268. Zu Bury, vgl. zuletzt Dönike, Martin (Hrsg.): Friedrich Bury, Briefe aus Italien an Goethe und Anna Amalia. Göttingen 2007. 
eine Kopie der Madonna della Sedia (1799-1815 im Louvre, heute Florenz), angefertigt von einem damals unbekannten jungen Maler aus Köln, dem zwanzigjährigen Carl Joseph Begas (1794-1854). ${ }^{27}$ Begas hatte zunächst ohne Auftrag an der Kopie gearbeitet. Und er war zu diesem Zeitpunkt bei weitem nicht der einzige junge Deutsche, der sich in der französischen Hauptstadt aus freien Stücken dieser reproduzierenden Tätigkeit nach Raffael widmete: die zeitweise in Paris lebenden Maler Christian Friedrich Tieck (1776-1851), ${ }^{28}$ Wilhelm Unger (1775-1855), ${ }^{29}$ Franz Gareis (1775-1803), ${ }^{30}$ Heinrich Kolbe (1771-1836), ${ }^{31}$ Ferdinand Jagemann (1780-1820), ${ }^{32}$ Gerhard von Kügelgen (1772-1820), ${ }^{33}$ Heinrich Olivier (1783-1848), ${ }^{34}$ Susanne Henry (1763-1819), ${ }^{35}$ Carl von Steuben (1788-1858), ${ }^{36}$ Ludwig Hummel (1770-1840), ${ }^{37}$ Christopher Wilhelm Eckersberg (1783-1853), ${ }^{38}$ der Däne Friedrich August von Klinkowström

27 Vgl. Müllejans-Dickmann, Rita / Haffner, Dorothee / Felbinger, Udo: Carl Joseph Begas (1794-1854). Blick in die Heimat. Heinsberg 1994, S. 107, Nr. 12, Abb. 8.

28 Vgl. Becker, Wolfgang: Paris und die deutsche Malerei. München 1971, S. 348, Anm. 653 ff. Zu Tieck vgl. Maaz, Bernhard: Christian Friedrich Tieck (1776-1851). Leben und Werk unter besonderer Berücksichtigung seines Bildnisschaffens, mit einem Werkverzeichnis. Berlin 1995.

29 Wilhelm Unger spezialisierte sich wohl auf Raffaelkopien in Aquarelle, vgl. Becker: Paris und die deutsche Malerei, S. 354 und Anm. $970 \mathrm{ff.}$

30 Gareis machte sich 1803 nach eigener Aussage mit „einer kleinen Kopie nach der Verklärung von Raffael [in Paris] einen Ruf". Vgl. Becker: Paris und die deutsche Malerei, S. 350 und Anm. $742 \mathrm{ff}$.

31 Heinrich Kolbe aus Düsseldorf kopierte ab 1803 die Heilige Familie Franz I, die Belle Jardinière und die Madonna della Sedia, vgl. Becker: Paris und die deutsche Malerei, S. 350 und Anm. $760 \mathrm{ff}$.

32 Ferdinand Jagemann, der zwischen 1802 und 1805 Kopien nach drei Madonnenbildern und dem Engel aus Heliodor malte, vgl. Becker: Paris und die deutsche Malerei, S. 351 und Anm. $817 \mathrm{ff}$.

33 Vgl. Becker: Paris und die deutsche Malerei, S. 352 und Anm. $878 \mathrm{ff}$. Kügelgen begann während seines Pariser Aufenthalts 1804 eine Kopie nach der Madonna della Sedia, in Dresden fertiggestellt.

34 Vgl. ebd., S. 355 und Anm. 990 ff. Heinrich Olivier kopierte 1808 die Johanna von Aragonien und eine Heilige Familie von Raffael.

35 Suzanne Henry kopierte 1814 die Belle Jardinière im Musée Napoléon, vgl. Savoy, Bénédicte (Hrsg.): Jean Henry, Journal d'un voyage à Paris en 1814. Paris 2001.

36 Steuben kopierte 1814 mindestens zwei Gemälde Raffaels im Louvre, die 1818 (Madonna del Pesce) und 1825 (La vierge au linge) von preußischen Königshaus erworben wurden, vgl. Becker: Paris und die deutsche Malerei, S. 352 und S. 409, Anm. $861 \mathrm{ff}$.

37 Vgl. Becker: Paris und die deutsche Malerei, S. 356 und S. 416, Anm. $1031 \mathrm{ff}$.

38 Vgl. ebd., S. 357 f. und S. 420, Anm. 1125 ff. 
$(1778-1835)^{39}$, von allen diesen Malern ist überliefert, daß sie in den ersten beiden Jahrzehnten des 19. Jahrhunderts in Paris Raffael kopierten. Und die Liste ließe sich sicherlich noch lange fortsetzen. Sie erzählt auf ihre Weise eine frühe Geschichte des kulturellen Raffaelkonsums und sie bedarf keines langen Kommentars: Gekoppelt mit der plötzlichen Veränderung der europäischen Raffael-Geographie zugunsten Frankreichs führte die Raffaelidolatrie der Deutschen spätestens ab 1800 geradezu zu einem nicht gesteuerten Boom der Kopieraktivität deutscher Maler in Paris. Dort stürzten sich fast alle - mit oder ohne Auftrag - auf ibren Raffael, so daß es übrigens fast lohnender wäre, eine Liste der Maler zu erstellen, die um 1800 Raffael nicht kopierten. Zugespitzt gesagt: Das Werk Raffaels ist in Deutschland (genauer: in Paris aber auf deutsche Initiative hin) innerhalb von einer Generation noch einmal, zweimal, dreimal neu erschaffen worden - nicht nur ideell eben, sondern auch „medienkonform“, piktural. Diese Aktivität muß übrigens so intensiv und sichtbar gewesen sein, daß sogar die Zeitschrift Paris, Wien und London im ersten Heft des Jahrganges 1811 berichtete: „Schon hat die Gemäldeausstellung [der Salon, B.S.] beinahe drei Monate gedauert, zum großen Ärger der Künstler, besonders der Teutschen, die in der Gallerie copiren, und denen mehrere in großen Unternehmungen unterbrochen sind." 40 Es scheint also sicherlich nicht übertrieben, die erste Phase der Produktion von Raffaelkopien und die damit verbundene erste Phase der Sammeltätigkeit solcher Kopien - ob in Berlin, Potsdam oder anderswo - als kollektives Unternehmen zu bezeichnen. Zumal sich zwischen Malern und Käufern auch eine ganze Reihe von Gutachtern, Beratern und Vermittlern bewegte, die auch vom Kopienfieber ergriffen waren, u.a. Aloys Hirt, Alexander [sic] und Caroline von Humboldt, Karl Friedrich Schinkel und Gustav Friedrich Waagen. ${ }^{41}$

Es versteht sich von selbst, daß diese Kopieraktivität der jungen, oft wenig bemittelten deutschen Künstler am Übergang von den Lehrjahren ins praktische Leben mit ganz konkreten beruflichen Hoffnungen verbunden war. Wer in der Lage war, Raffael (gut) zu kopieren, konnte sich im großen Pariser Konkurrenzgetümmel der Jahre um 1800 eine gewisse Sichtbarkeit erarbeiten - so soll zum Beispiel eine Kopie der Belle Jardi-

39 Vgl. ebd., S. 356 und S. 418, Anm. $1066 \mathrm{ff}$.

40 N.N.: „Etwas über die Pariser Gemäldeausstellung von 1810-11“, in: Paris, Wien und London, 1/1811, S. 227-231, hier: S. 228.

41 S.o., Anm. 13. 
nière den Maler François Gérard veranlaßt haben, Heinrich Kolbe in sein Atelier aufzunehmen. ${ }^{42}$ Auch nach außen hin, nach Deutschland etwa, waren Raffael-Kopien ein sicheres Mittel, Kunstfertigkeit zu demonstrieren - im Jahre 1804 zum Beispiel beteiligte sich Ferdinand Jagemann mit vier in Paris entstandenen Raffael-Kopien an der Ausstellung der Weimarer Kunstfreunde. ${ }^{43}$ Darüber hinaus hatten viele junge Maler die begründete Hoffnung, mit solchen Reproduktionen in Öl lukrative Geschäfte mit privaten Käufern zu machen.

Denn es gab einen Markt für diese Raffael-Kopien, nicht nur am preußischen Hof und übrigens nicht nur erst seit der gewaltsamen Vereinigung seines Werks in Paris. Über den auf Sepiakopien spezialisierten Maler Jakob Crescenz Seydelmann (1750-1829) schrieb bereits zu Beginn der 1790er Jahre ein Besucher der Dresdner Gemäldegalerie:

Die Engländer, Russen und Polen sehen kein Geld an, etwas von ihm zu bekommen, und Seydelmann gehört mit unter die glücklichen und zufriedenen Menschen, die man so selten unter Männern von großen Talenten, antrifft. Ich sahe eine Copie von der Madonna nach Raphael, für die ihm die Fürstin Radzivil fünfhundert Reichsthaler bezahlte. ${ }^{44}$

Neu allerdings scheint ab 1800 die Tatsache gewesen zu sein, daß sich Raffaelkopien in Farbe verbreiteten - ein Umstand, der sich vielleicht mit der besonders liberalen Kopierpolitik im Musée Napoléon erklären läßt. ${ }^{45}$ Schenkt man nämlich den Worten Gottfried Seumes Glauben, so war es zum Beispiel in Dresden nicht möglich, um 1800 ganze Gemälde in Öl zu kopieren: „Kein Künstler“, so schrieb er, „darf auf der Galerie ein Stück ganz fertig kopieren, wie man mich versichert hat. Dies zeigt eine sehr kleinliche Eifersucht. [...] Auch darf kein Maler länger als die bestimmten zwei Stunden oben arbeiten, welches für die Kopisten in Öl eine Zeit ist, in welcher fast nichts gemacht werden kann". ${ }^{46}$ Im Kopienreglement der Dresdner Galerie aus dem Jahre 1858 wird sogar das Kopieren der Sixtinischen Madonna (neben drei weite-

42 Vgl. Becker: Paris und die deutsche Malerei, S. 350 und Anm. 768

43 S.o., Anm. 32.

44 Bozenhard, Emanuel: Bemerkungen auf einer Reise von Kopenhagen nach Wien im Jahr 1793. Hamburg 1795, S. 55-60, abgedruckt in: Savoy (Hrsg.): Tempel der Kunst, S. 428.

45 Vgl. Cuzin, Jean-Pierre / Dupuy, Marie-Anne (Hrsg.): Copier-Créer. De Turner à Picasso: 300 auvres inspirées par les maîtres du Louvre. Ausstellungskatalog, Musée du Louvre. Paris 1993.

46 Seume, Johann Gottfried [1801]: Spaziergang nach Syrakus im Jabre 1802. Hrsg. von Albert Meier. München 2002, S. 6. 
ren Werken) $)^{47}$ ausdrücklich untersagt - ein Indiz für ihre andauernde Anziehungskraft und für die damit verbundene Nachfrage nach solchen Kopien.

Trotz der lückenhaften Quellenlage lassen sich also bereits für die Zeit vor 1815, neben dem preußischen Königshaus, eine Reihe weiterer Sammler und Käufer von Raffaelkopien aus dem Louvre identifizieren sicherlich nur ein Bruchteil vom tatsächlichen Kundenkreis: 1810 kaufte Metternich eine Kopie der Belle Jardinière von Klinkowström. ${ }^{48}$ Der Herzog von Oldenburg gab im Winter 1808/09 eine Kopie der Heiligen Familie bei L. Hummel im Auftrag. ${ }^{49}$ Die vermutlich 1800 entstandene Kopie der Madonna della Sedia von Tieck hing laut A. Raczynski Anfang der 1840 er im Humboldt-Schloß in Tegel. ${ }^{50}$ Die spezielle Dynamik, die hier zwischen dem Angebot Dutzender von Malern und den potenziellen Käufern zustande kam, wird in einem Brief der bereits erwähnten Therese aus dem Winckel anschaulich beleuchtet. Im Januar 1809 bat die finanziell bedrängte junge Frau von Paris aus ihren Freund Karl August Böttiger in Dresden zu ermitteln,

ob es nicht möglich sey, von irgend einem Fürst oder einer Fürstin [...] so als Hofmalerin angestellt zu werden, daß [sie] die ausgewähltesten Gemälde aus den Gallerien in Frankreich, Deutschland und Italien für sie kopierte und sie so mit geringen Kosten die seltensten Werke ihrer Lieblingsmeister in täuschend treuen Nachbildungen um sich her versammeln könnten. ${ }^{51}$

Die alten Meister im Salon - daß das Hängen solcher Kopien in Privaträumen ein Ausdruck für die ästhetische Positionierung und kunstreligiöse Gesinnung ihrer Eigentümer war, muß nicht lange erörtert

47 „Reglement, die Vorschriften für das Copiren der in der königlichen GemäldeGalerie befindlichen Gemälde betreffend: [...] Außerdem wird auch das Copiren folgender Gemälde, als: a) die Sixtinische Madonna von Rafael, b) die Madonna von Holbein, c) der Zingroschen von Tizian, d) die Heilige Nacht von Correggio, nicht gestattet. Die Erlaubnis hierzu kann allein von seiner Majestät dem Könige ertheilt werden; diesfallsige Gesuche werden nur von ausgezeichneten Künstlern angenommen werden. "Zitiert nach Strittmatter: Das, Gemäldekopieren', S. 288-290, hier: S. 289.

48 S.o., Anm. 35.

${ }^{49}$ S.o., Anm. 34.

50 "Seine damals nach der madonna della Sedia gefertigte Zeichnung, in der Größe des Urbildes, befindet sich in Tegel, und ist ein sehr ausgezeichnetes Werk", Raczynski, A.[thanasius]: Geschichte der neueren deutschen Kunst. 3 Bde. Berlin 1836-41, Bd. 3, 1841, S. 190.

51 Briefe der Therese aus dem Winckel an Karl August Böttiger, Paris, 29. Januar 1808, abgedruckt in: Strittmatter: Das, Gemäldekopieren', S. 304 f., hier: S. 304. 
werden, man denke nur an die Kopiensammlung im Wörlitzer Schloßensemble (nach Werken Tizians, Renis, Domenichinos, Caraccis aber auch Holbeins, Cranachs, Rubens' und van Dycks) oder an die 50 Kopien vor allem nach italienischen Meistern (darunter auch Raffael), die im Gut Emkendorf bei Kiel, diesem Mittelpunkt eines geistig-religiösen Gelehrtenkreises um Friedrich Karl Reventlow, von ca. 1800 bis 1926 hingen. ${ }^{52}$

\section{Vom Wohnzimmer ins Museum}

Eine systematische Untersuchung über die Präsenz von originalgroßen Kopien nach Raffael in europäischen Interieurs der ersten Jahrzehnte des 19. Jahrhunderts steht aus. Aber alleine schon anhand einer kleinen Auswahl bekannter Innenraumdarstellungen entsteht der Eindruck einer unerhörten Fülle an solchen Kopien, zumindest - die Quellenlage ergibt dieses vorläufige Bild - in fürstlichen Gemächern des deutschsprachigen Raums und in Rußland, wobei auch adlige und bürgerliche Interieurs zum Beispiel in England eine Untersuchung verdienten. In Rußland, wo die Tradition der Raffaelkopien als Teil eines Ausstattungsprogramms bereits von Katharina II. eingeführt worden war (die Kopien der Loggien-Fresken in der Eremitage entstanden um 1780, die von Georg Velten entworfene Stanza di Raffaello in Carskoe Selo im Jahr 1779), ${ }^{53}$ wo darüber hinaus das deutsche Element durch die Heiratspolitik des Zarenhauses sehr präsent war, spielten Raffaelkopien um 1800 offensichtlich eine wichtige Rolle in vielen Palästen. Auf einem Aquarell aus diesen Jahren ist z.B. das Kabinett der Gattin Alexanders I. von Rußland, Elisabeth Alexejewna (Luise von Baden), im Petersburger Winterpalais zu sehen

Ein einziges, schlicht gerahmtes Bild ziert den Raum: Die sixtinische Madonna. Ein weiteres Aquarell von Wilhelm Barth aus dem Jahre 1823 zeigt die Intimsphäre der preußischen Kronprinzessin Charlotte, der

52 Vgl. Strittmatter: Das, Gemäldekopieren', S. 309.

53 Zwischen 1778 und 1785 stellte Christoph Unterberger für Katharina II. originalgroße Kopien von Raffaels Loggien-Fresken in enkaustischer (Wachs-) Malerei her. Dies war zugleich ein wichtiger Versuch der Neubelebung einer antiken Technik, um die sich gleichzeitig mehrere Maler bemühen. Vgl. Frank, Christoph: "L'arte e l'architettura romane nella corrispondenza di Caterina II di Russia“, in: Nicola Navone (Hrsg.): Dal mito al progetto. La cultura architettonica dei maestri italiani e ticinesi nella Russia neoclassica. Mendrisio 2004, S. 60-77. 


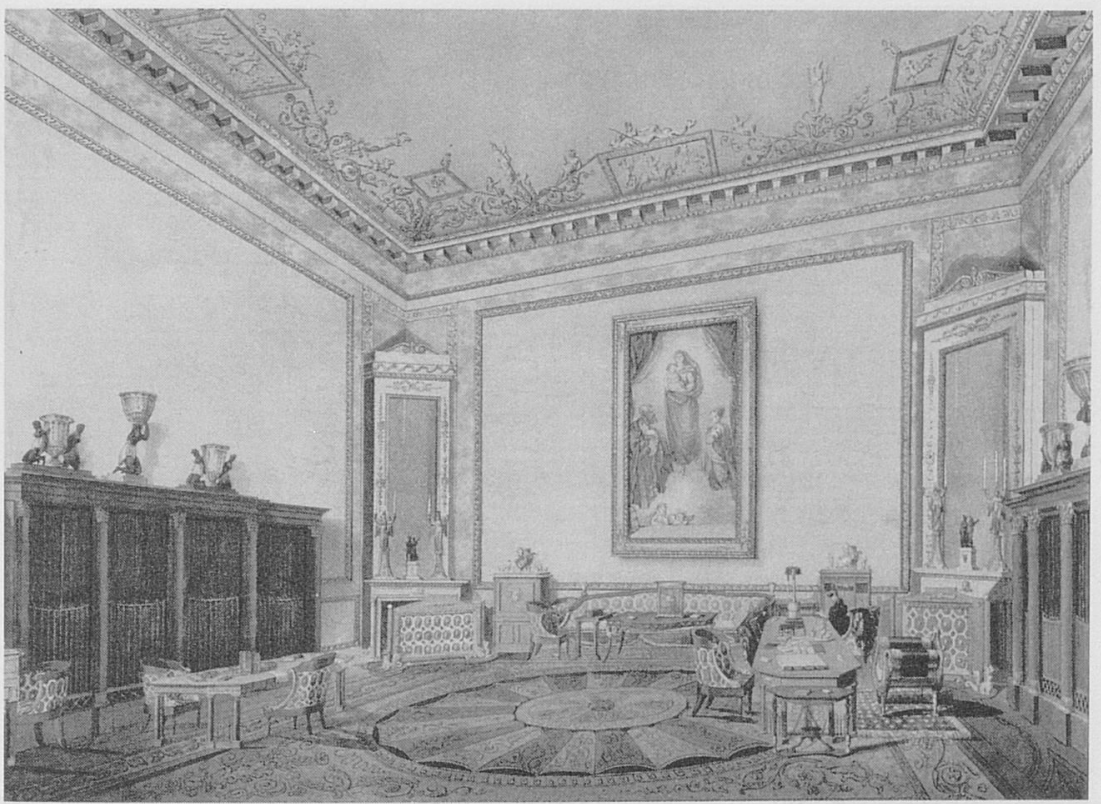

Abb. 6: Kabinett der Gattin Alexanders I. von Rußland, Elisabeth Alexejewna (Luise von Baden), im Petersburger Winterpalais, um 1809

verheirateten Großfürstin Alexandra Fjodorowna und späteren Zarin von Rußland im Anitschkow-Palais, ebenfalls in Sankt Petersburg. ${ }^{54}$

An der Stirnwand des kleinen Raums hängen die Engel der Sixtinischen Madonna, rechts in der unteren Reihe, nur schemenhaft zu erkennen, eine Kopie der Madonna Conestabile in ihrem charakteristischen Schinkelrahmen. Weitere Untersuchungen würden sicherlich noch mehr Beispiele dieser Art zutage fördern.

In Berlin und Potsdam war die wachsende Sammlung der in Paris und Italien beauftragten Raffaelkopien, die dann ab 1858 im Raffaelsaal zum öffentlichen Gebrauch versammelt wurden, zunächst in verschiedenen Wohnungen und Palais verteilt. Ikonographisch dokumentiert ist die Präsenz von Reproduktionen nach Raffael unter anderem im Arbeitszimmer Friedrichs Wilhelms III. im Kronprinzenpalais, im grünen

${ }^{54}$ Barth, Wilhelm: Zimmer der Großfürstin Nikolai im Anitschkow-Palais, St. Petersburg. Potsdam, Stiftung Preußische Schlösser und Gärten Berlin-Brandenburg, Plankammer, Aquarellsammlung, abgebildet in: Roenne: Ein Architekt rabmt Bilder, S. 115 . 


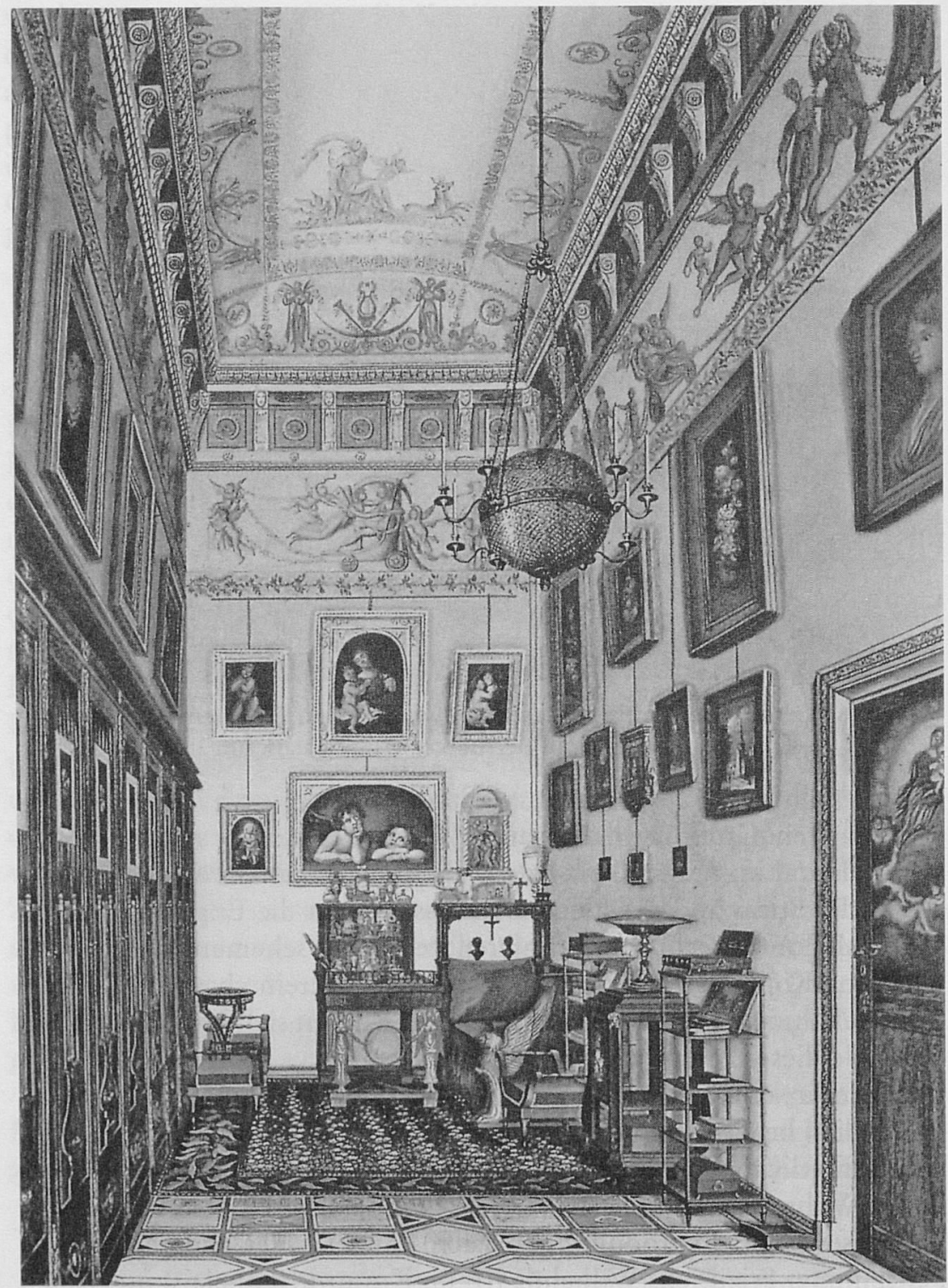

Abb. 7: Wilhelm Barth: Zimmer der Großfürstin Nikolai im Anitschkow-Palais, St. Petersburg, 1823 


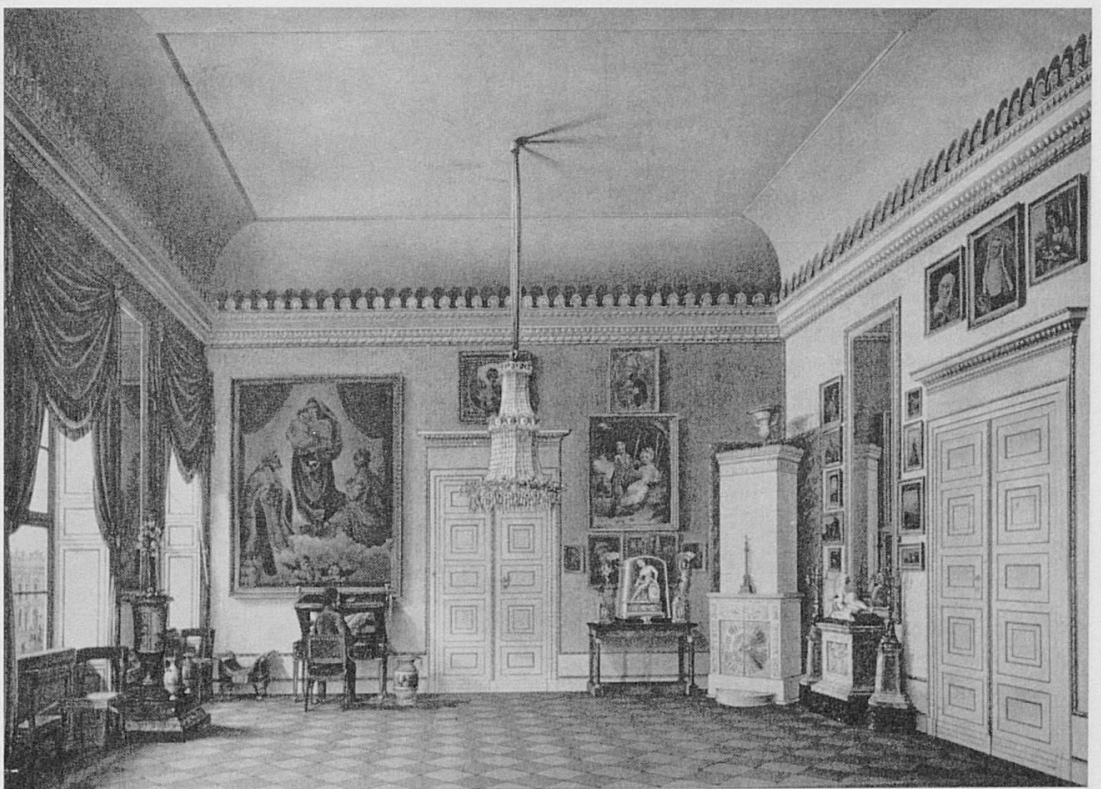

Abb. 8: Arbeitszimmer König Friedrich Wilhelms III, Kronprinzenpalais, Unter den Linden, Druck

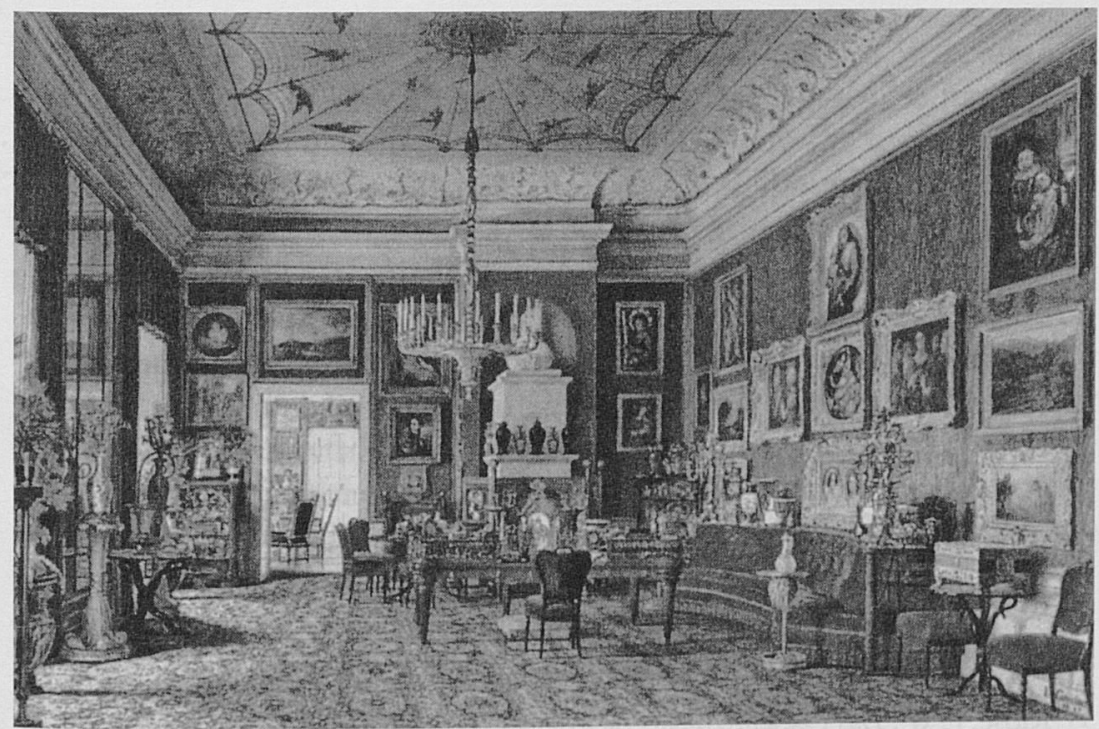

Abb. 9: Eduard Gaertner: Grünes Zimmer in der Wobnung des Prinzen Wilhelm und der Prinzessin Marianne, 1847 


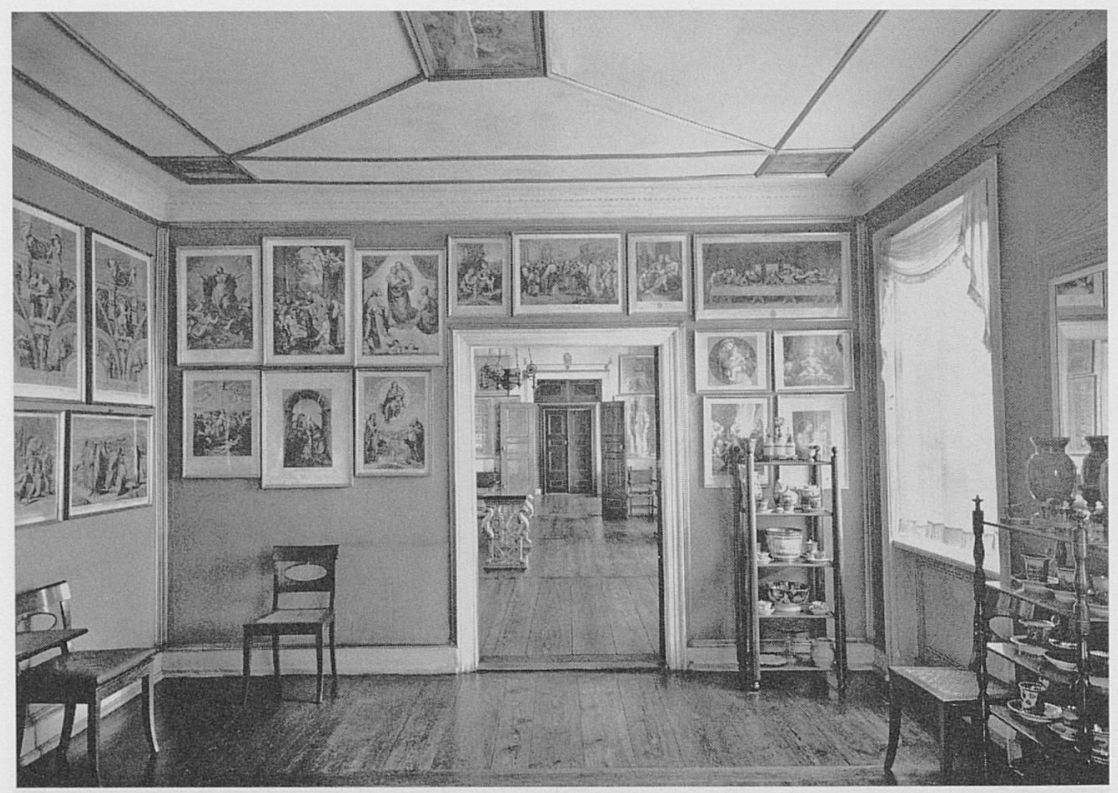

Abb. 10: Park Sanssouci, Schloß Charlottenhof, Schinkel K. L., Persius L., Innenansicht, Durchblick zum Speisesaal, Aufnahme Walter Möbius 1955

Zimmer der Wohnung des Prinzen Wilhelm und der Prinzessin Marianne im Berliner Schloß - sowie in Charlottenhof zu Potsdam, wo einerseits eine Fülle von Stichen nach Raffael und andererseits eine Teilkopie der Transfiguration in Sepia von Seydelmann hingen. Ein Entwurf von Karl Friedrich Schinkel deutet auch auf Pläne für ein sogenanntes „Raffaelzimmer" im Pavillon Friedrich Wilhelm III. zu Charlottenburg hin.

Besonders aufschlußreich in diesem Zusammenhang ist aber vor allem ein Aquarell von Friedrich Wilhelm Klose aus dem Jahre 1840, das das sogenannte Chamois-Zimmer im Berliner Kronzprinzenpalais darstellt. ${ }^{55} \mathrm{Zu}$ sehen sind, dicht an dicht und übereinander gehängt, vierzehn Gemäldekopien, ausnahmslos nach Raffael. Überraschend an dieser Ansicht sind weniger die Gemälde selbst - aus anderen Quellen ist

55 Klose, F. W.: Das Chamoiszimmer im Königlichen Palais zu Berlin, um 1840. Potsdam, Stiftung Preußische Schlösser und Gärten Berlin-Brandenburg, Plankammer, Aquarellsammlung, abgebildet in: Bussler: Der Rafael-Saal, 1861, S. XXVII. 


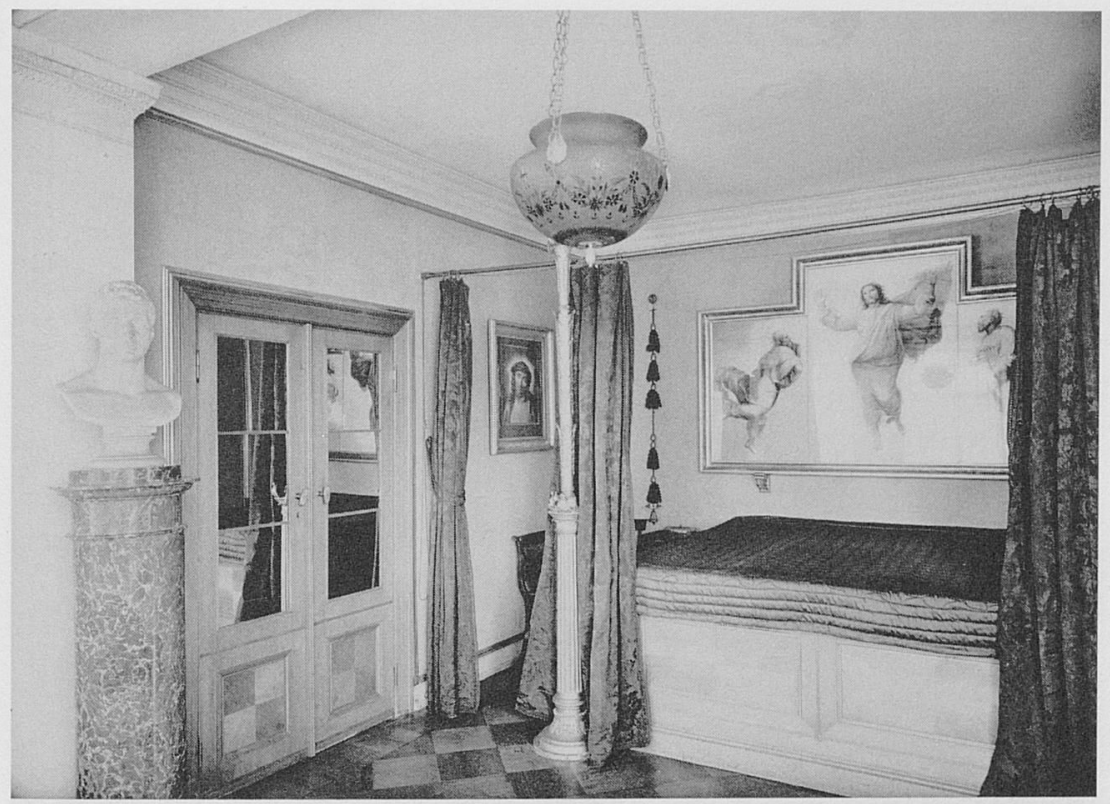

Abb. 11: Park Sanssouci, Schloß Charlottenhof, Scbinkel K. L., Persius L., Innenansicht, Schlafzimmer, Aufnahme Walter Möbius 1955

bekannt, daß dieses Zimmer die wachsende Sammlung der in Paris und Italien beauftragten Kopien versammelte, die wir später im Raffaelsaal wiederfinden ${ }^{56}$ - als die Tatsache, daß vor den Raffael-Kopien mehrere Tische aufgestellt sind, auf denen sieben aneinander gereihte Käfige stehen, bewohnt jeweils von einem Papagei. Über die akustische Atmosphäre in einem solcher Art bevölkerten Zimmer kann man nur spekulieren. Man kann sich aber des merkwürdigen Eindrucks nicht erwehren, daß die sieben Papageienkäfige mit den sieben unmittelbar dahinterhängenden Raffaelkopien korrespondierten. Der „Heiland der Farbe" an der Stubenwand und die exotische Farbenpracht der Papageien im Käfig - hier korrespondieren zwei Formen der Domestizierung, die für das 19. Jahrhundert typisch sind und offensichtlich verwandter sind, als man es zunächst glauben mag.

Das Chamois-Zimmer im Kronprinzenpalais vereinigte also bis zur Eröffnung des Raffael-Saals im Herbst 1858 einen beträchtlichen Teil

56 Vgl. Bartoschek, Gerd in: ebd., S. VI. 


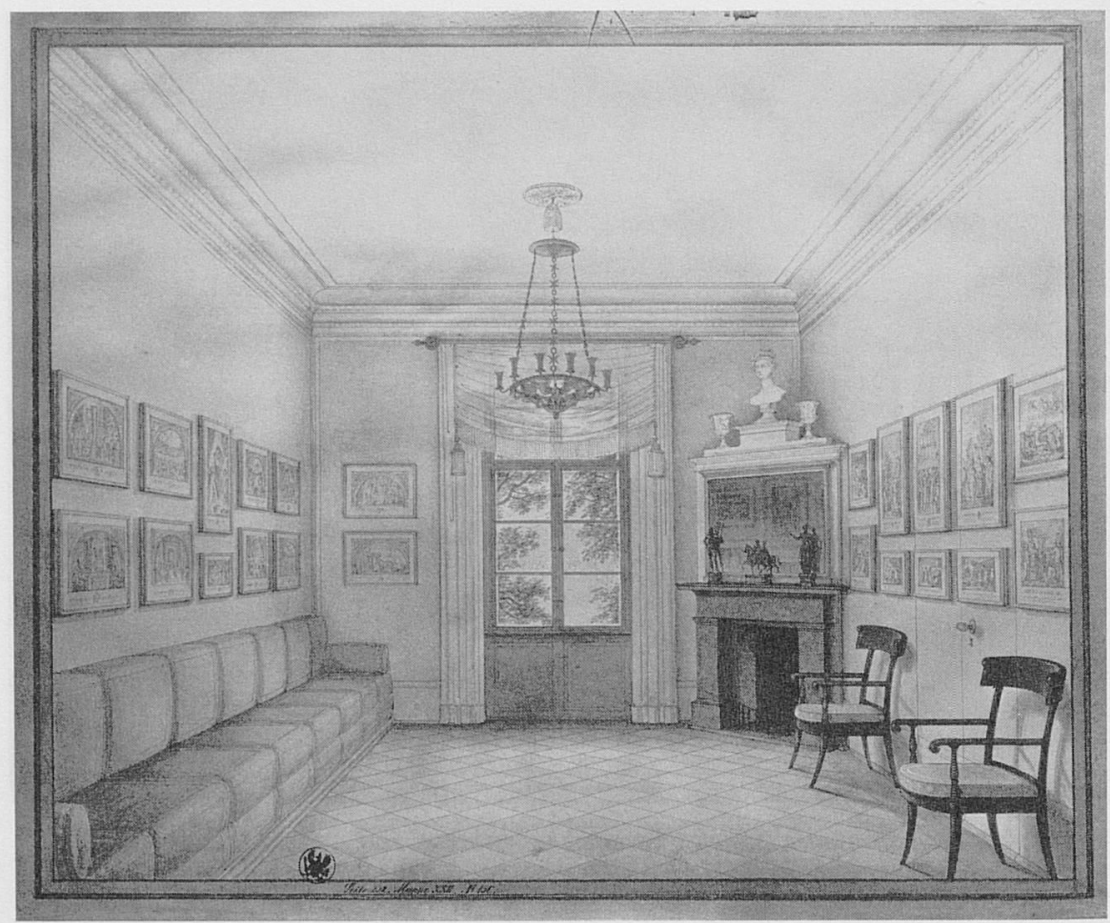

Abb. 12: Karl Friedrich Schinkel: Das Raffaelzimmer. Entwurffür das Raffaelzimmer im Pavillon Friedrich Wilhelm III. zu Charlottenburg, Aquarell

der seit 1804 am Preußischen Hof gesammelten Raffaelkopien. Auf die ersten punktuellen Erwerbungen folgte nämlich in den Jahren bis ca. 1825 die Vergabe zahlreicher mit Stipendien verbundenen Kopienaufträge durch das preußische Königshaus, eine Form der Künstlerförderung, die eng mit dem Projekt einer Erneuerung der lokalen Malerschule im Geiste Raffaels verbunden war. ${ }^{57}$ Das bekannteste Beispiel für ein solches Stipendium ist dasjenige, das Wilhelm Hensel 1823 vom preußischen König bekam, um in Rom unter anderem eine Kopie der Transfiguration herzustellen (1828 fertiggestellt). ${ }^{58}$ Über den Rhythmus der Erwerbungen von Raffaelkopien am preußischen Hofe schrieb der bereits zitierte Robert Bussler rückblickend:

57 Vgl. u.a. Raczynski, A.: Geschichte der neueren deutschen Kunst, Bd. 3.

58 Vgl. Bussler: Der Rafael-Saal, 1861, S. 55 f. 


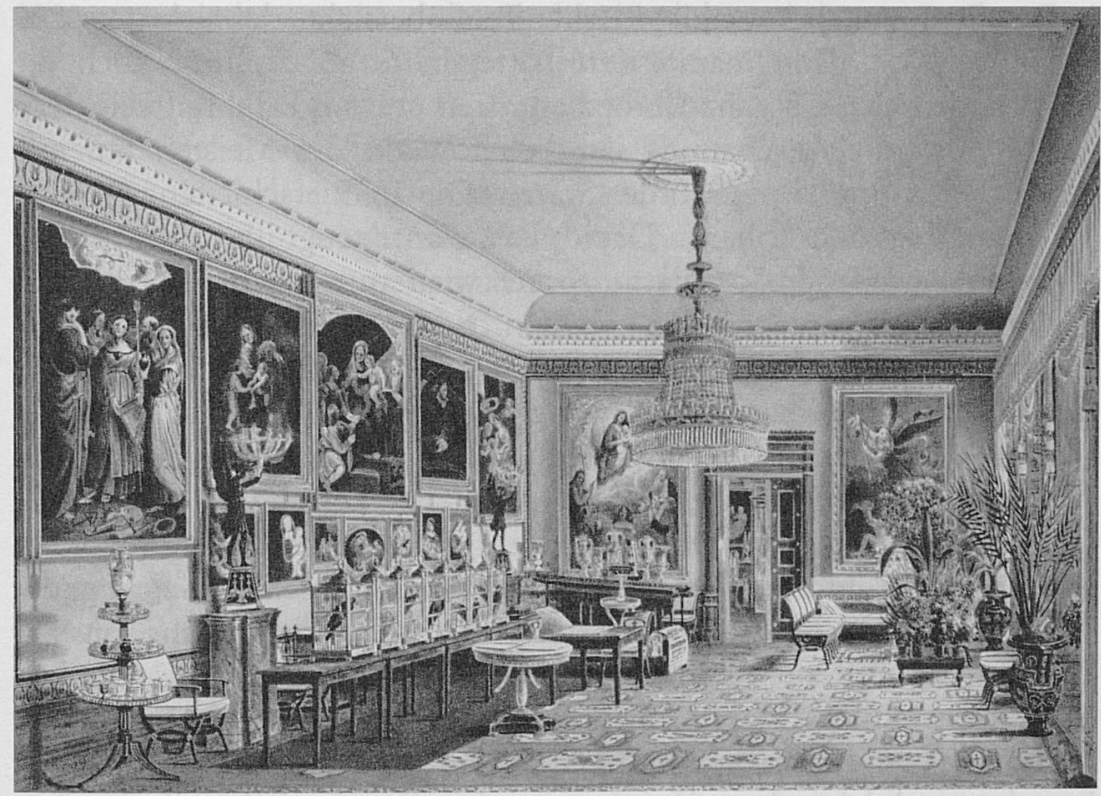

Abb. 13: F. W. Klose: Das Chamoiszimmer im Königlichen Palais zu Berlin um 1840

Die erste Gruppe der Erwerbungen bilden die [1814 in Paris, B.S.] gewonnenen sechs Acquisitionen, während die zweite Gruppe von den in [Friedrich Wilhelms, B.S.] segensreicher Regierung ferner angekauften und bestellten Copien nach Raffael gebildet wird. Von den erlauchten Nachfolgern wurden dann systematisch die wesentlichen Lücken ausgefüllt und so allmählig bis zu den früheren Werken des großen Meisters zurückgegriffen. ${ }^{59}$

\section{Kopienmuseum und Fotografie}

Zunächst bestand in Berlin also keinerlei Absicht, die vielen für die privaten Räume der königlichen Familienmitglieder gekauften Raffaelkopien an einem einzigen Ort zu vereinigen, geschweige denn ihnen eine öffentliche Sichtbarkeit mit geschmacksbildnerischer Absicht zu verschaffen. Der Weg ins Museum war nicht vorgesehen. Deswegen ist er auch besonders interessant. Was passierte um 1850? Drei Phänomene mit weitreichenden museumsgeschichtlichen Folgen seien hier besonders hervorgehoben:

59 Bussler: Der Rafael-Saal, 1866, S. 20. 
1. Der Drang nach kunsthistorischer Totalübersicht, der sich um 1800 im Musée Napoléon manifestiert hatte, hatte ab ca. 1830 auch die Kunstwissenschaft in ganz Europa ergriffen und im Falle Raffaels dazu geführt, daß die Anschauung sämtlicher Werke des Meisters zur methodischen Grundlage geworden waren. Die 1839 erschienene RaffaelMonographie von Johann David Passavant zeugt davon. ${ }^{60}$ Dieser medienübergreifende Totalitätsanspruch wiederum brachte für die $\mathrm{Mu}$ seumswelt ein Utopieproblem mit sich: Die Unmöglichkeit der Vereinigung authentischer Werke Alter Meister an einem einzigen Ort. Francis Haskell hat wunderbar gezeigt, wie genau in den 1850er Jahren dieses Utopieproblem unter dem entscheidenden Impuls des Berliner Kunsthistorikers Gustav Friedrich Waagen und der Leitung eines weiteren deutschstämmigen Kunstexperten, George Scharf, in Großbritannien gelöst wurde: Durch die Erfindung einer neuen musealen Gattung, der ephemeren Ausstellung Alter Meister, einer Erfindung, die übrigens zur Politisierung und zur Professionalisierung des Ausstellungswesens beitrug. Als wichtigste Etappe auf dem Weg zur Blockbuster-Ausstellung, wie wir sie heute kennen, nennt Haskell die stark von kunsthistorischer Forschung, didaktischen Absichten und nationaler Rhetorik geprägte „Art Treasures Exhibition of the United Kingdom“ in Manchester (1857), die Holbein-Ausstellung in Dresden (1871), die patriotismusschwangere Michelangelo-Schau in Florenz (1875) sowie die Rembrandt-Ausstellung in Amsterdam (1898), zu der erstmals ein begleitender kunsthistorischer Fachkongreß tagte. Es lohnt sich aber auch, die sehr frühe, bezeichnenderweise ebenfalls von G.F. Waagen organisierte, 1848 in Berlin gezeigte Raffael-Ausstellung in diese Reihe aufzunehmen. ${ }^{61}$

Worum handelte es sich bei dieser im revolutionären Monat März 1848 in der Rotunde des Schinkelschen Museums am Lustgarten eröffnete Schau? Sie sollte, so hieß es im Katalog, für einige Wochen einen Überblick über „die künstlerische Entwicklung Raphaels in ihrer ganzen Stufenfolge" bieten. ${ }^{62}$ Dementsprechend zeigte sie ein breites Spektrum an Werken und Werkgattungen, neben allen Gemäldekopien nach Raffael aus den königlichen Gemächern auch Kupferstiche, einige Originalzeichnungen sowie die 1844 für das Museum erworbenen Raffael-Teppiche. Sie war in gewisser Hinsicht eine museale, räumlich erfahrbare

60 Vgl. dazu Schröter: Raphael-Kult; Osterkamp: Raffael-Forschung.

${ }^{61}$ Haskell, Fancis: The Ephemeral Museum. Old Master Paintings and the Rise of the Art Exbibition. New Haven/London 2000.

${ }^{62}$ Waagen, Gustav Friedrich: Verzeichnis der Copien, S. 4. 
Übersetzung des neuen methodischen Bedürfnisses nach Gesamtübersicht. Gustav Friedrich Waagen hatte sich das ,Event ${ }^{6}$ ausgedacht und den Katalog dazu verfaßt. Bemerkenswert ist die Symbolik der Daten: Diese Raffael-Ausstellung eröffnete ihre Tore anläßlich des 600. Jahrestages der Grundsteinlegung des Kölner Doms. Während Bayern zu diesem Anlaß fünfleuchtende Fenster im Stil der Nazarener für das südliche Seitenschiff der weiterzubauenden Kathedrale stiftete (die sogenannten „Bayernfenster"), ${ }^{63}$ inszenierte man in Berlin das leuchtende Gesamtwerk Raffaels als patriotisch-religiöse Angelegenheit, da ja die zu erwartenden Einnahmen der Ausstellung in einen Fonds für den als nationale Aufgabe empfundenen und propagierten Weiterbau des Kölner Doms fließen sollten - eine Tradition der patriotischen Inanspruchnahme von Kunstausstellungen, die übrigens schon während der Befreiungskriege in Berlin erprobt worden war. ${ }^{64}$ Raffael im Museum zwischen Nation, Religion, Kunstwissenschaft und Politik - besser als an diesem Beispiel läßt sich die funktionsgeschichtliche Verankerung des deutschen Raffaelkultes des 19. Jahrhunderts (und somit des Potsdamer Raffaelsaals, der eine direkte Frucht dieses frühen Experimentes gewesen ist) nicht schildern.

2. Eine weitere museumsspezifische Antwort auf das neue Bedürfnis der Kunstwissenschaft nach Totalübersicht des CEuvres alter Maler ist die Erfindung und schnelle Verbreitung von sogenannten Kopienmuseen, ebenfalls ab ca. 1850. Diese Erfindung löste intensive Diskussionen aus und initiierte viele nicht ausgeführte Projekte, die es verdienten, systematisch und transnational untersucht zu werden. Auch hier kam das Streben nach einer vollständigen Repräsentation der kunstgeschichtlichen Entwicklungen zum Ausdruck, auch hier entstanden in ganz Eu-

${ }^{63}$ Vgl. Dahmen, Stephan: Die Bayernfenster des Kölner Doms 1844-1848: Kirchenausstattung zwischen Kunst, Theologie und Politik. Köln 2008. Zum Gesamtkomplex vgl. Nipperdey, Thomas: „Der Kölner Dom als Nationaldenkmal“, in: Ders.: Nachdenken über die deutsche Geschichte. Essays. München 1986, S. 189-207.

${ }^{64}$ Vgl. zum Beispiel im Jahre 1814 N.N. (Hrsg.): Verzeichniss von Kunst- und LiteraturWerken welche zum Besten Verwundeter öffentlich ausgestellt und dargelegt, zum Theil auch geschenkt und käuflich sind, Berlin 1814; des Weiteren 1815 N.N. (Hrsg.): Verzeichnis von Gemälden und Kunstwerken, welche durch die Tapferkeit der vaterländischen Truppen wieder erobert worden und auf Verfügung eines Hohen Ministerii des Innern in den Sälen der königl. Akademie der Künste zu Gunsten der verweundeten Krieger des Vaterlandes [...] öffentlich ausgestellt sind. Berlin 1815. Vgl. Savoy: Patrimoine annexé. Bd. 1, S. 395 . 
ropa Institutionen, die heute in Vergessenheit geraten sind aber als kunstdidaktisches und kunstwissenschaftliches Schlüsselphänomen des Historismus Aufmerksamkeit verdienen. In all diesen Kopienmuseen und -galerien spielte das Werk Raffaels eine Schlüsselrolle: Das eindrucksvollste und mit dem Potsdamer Raffaelsaal am nächsten verwandten Beispiel für diese Entwicklung liefert ohne Zweifel der seit Mitte des 19. Jahrhunderts „Raffaelsaal“ genannte Kopiensaal in der Akademie der Künste in Sankt Petersburg. ${ }^{65}$ Dort hingen (und hängen heute noch) sehr frühe, originalgroße Kopien der Raffaelfresken in den Stanzen, die bereits in den 1820 er und 1830 er Jahren von russischen Stipendiaten der Akademie in Rom, so zum Beispiel von Karl Briullov (1799-1852) angefertigt worden waren und Mitte des 19. Jahrhunderts in eine Galerie des Akademiegebäudes am Neva-Ufer zu Studienzwecken relativ niedrig gehängt wurden - eine Sammlung, die seit den 1830er Jahren kontinuierlich um Kopien nach Einzelwerken Raffaels erweitert wurde. Ein weiteres Beispiel für ein frühes Kopienmuseum bildet die Sammlung Bernhard Augusts von Lindenau (1848), die auf dem Pohlhof in Altenburg 36 Werke nach Raffael unter insgesamt 131 Kopien vereinigte; 66 darüber hinaus die berühmte, heute teilweise erhaltene Schack-Galerie in München (1874), die unter 85 Kopien 6 Werke nach Raffael zeigte; 67 das kurzlebige und dennoch hochinteressante Musée des Copies von Charles Blanc in Paris (1873), ${ }^{68}$ das als Höhepunkt dieser institutionellen Entwicklung betrachtet werden darf, präsentierte 42 Raffaels unter etwa 150 Kopien; und das verhältnismäßig späte Reiff-Kopienmuseum in Aachen (1908), wo der Schwerpunkt zwar auf den niederländischen Landschafts- und Genreszenen des 17. Jahrhunderts lag, wo aber zu Beginn des 20. Jahrhunderts noch sechs Kopien nach Raffael zum Programm gehörten. Interessanterweise stand also der 1858 eröffnete Raffaelsaal in der Orangerie zu Potsdam ganz am Anfang einer museographischen Bewegung, die erst zu Beginn des 20. Jahrhunderts in Frage gestellt wurde. Die doppelte Singularität des Potsdamer Beispiels springt allerdings ins

65 Тютрина, Елена: Парадные залы академии художеств, Российская академия художеств - научко-исследовательский музей. Sankt Petersburg 2004 [Tjutrina, E. V.: Paradnyje zaly akademii xudožestv, Rossijskaja akademija xudožestv - naučnoissledovatel'skij muzej. Sankt Petersburg 2004].

66 Vgl. Strittmatter: Das, Gemäldekopieren; S. 311.

67 Vgl. Ruhmer, Eberhard (Hrsg.): Schack-Galerie. Vollständiger Katalog. 2 Bde. München 1969.

68 Vgl. zuletzt Scherkl, Robert: „Charles Blancs Musee des copies. Kopien wie Originale?", in: Zeitschrift für Kunstgeschichte, 63/2000, 3, S. 358-371. 


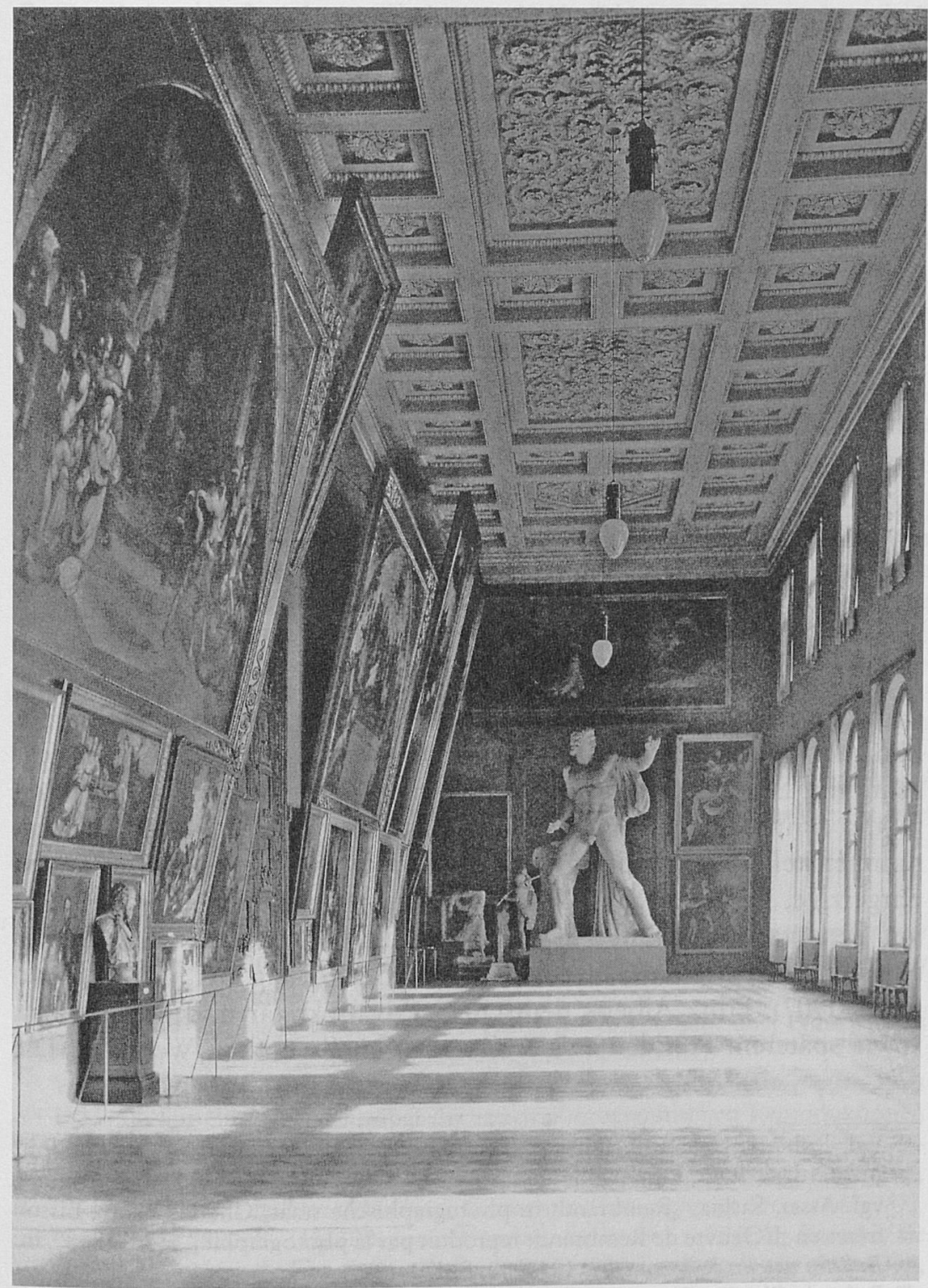

Abb. 14: Raffaelsaal (Kopiensaal) in der Akademie der Künste in Sankt Petersburg 
Auge: In Potsdam wurde Raffael nicht kunsthistorisch eingeordnet sondern als a-historisches Phänomen, als Unikum inszeniert. Und die Inszenierung diente nicht unmittelbar der Ausbildung junger Künstler, war doch der Raffaelsaal in der Orangerie (im Gegensatz z. B. zum Petersburger Raffaelsaal) weder strukturell noch topographisch mit einer Ausbildungsstätte verbunden.

3. Ein drittes Phänomen ist eng verbunden sowohl mit den methodischen Entwicklungen der kunstwissenschaftlichen Forschung ab dem zweiten Drittel des 19. Jahrhunderts als auch mit der Historiographie des Werkes Raffaels und der Problematik der Reproduktion von Kunstwerken: Die Erfindung der Fotografie. Auch sie spielt eine zentrale Rolle für das Verständnis des Raffaelsaals in Potsdam, ja die zentrale Rolle. Es ist hier nicht der Ort, die frühe, spannende und transnationale Geschichte der fototechnischen Reproduktion von Kunstwerken und ihrer viel diskutierten Ausstellung in Museen zu beleuchten. ${ }^{69}$ Ich beschränke mich auf einige Bemerkungen, die in unmittelbarer Verbindung mit dem Raffaelsaal stehen.

Während sich das preußische Königshaus in den 1850er Jahre Gedanken zur angemessenen Präsentation der bunten Raffaelkopien aus königlichem Besitz machte, beschäftigte sich in England der deutschstämmige Ehemann der Königin Victoria, Albert von Sachsen-CoburgGotha (Prinz Albert) bis zu seinem Tode im Jahre 1861 mit der Zusammenstellung eines fotografischen Corpus des Werkes Raffaels. Das ehrgeizige, heute etwas in Vergessenheit geratene Projekt stützte sich auf dem von Passavant zusammengestellten catalogue raisonné des Meisters, die wissenschaftliche Verantwortung lag bei Carl Ruland (1834-1907), Prinz Alberts deutschem Privatsekretär und Bibliothekar sowie späterem Präsidenten der Goethe-Gesellschaft in Weimar. ${ }^{70}$ Die

${ }_{69}$ Vgl. Roberts, Helene E. (Hrsg.): Art History through the Camera's Lens. London/ Amsterdam 1995; zum Verhältnis zwischen Kopienmuseen und Photographie, vgl. Asser, Saskia: „Rembrandt in photographische staat: Charles Blanc, Bisson frères en ,L'Oeuvre de Rembrandt reproduit par la photographie, '1853-1858“, in: Bulletin van het Rijksmuseum, 48/2000, S. 170-199.

70 Ruland, Charles (Hrsg.): The works of Raphael Santi da Urbino as represented in the Raphael collection in the Royal Library at Windsor Castle, formed by H. R. H. the Prince Consort, 1853-1861 and completed by Her Majesty Queen Victoria. Weimar 1876. Vgl. Montagu, Jennifer: „The ,Ruland / Raphael Collection, Visual Resources“", abgedruckt in: Roberts (Hrsg.): Art History through the Camera's Lens, S. 37-57. Zu Carl Ruland, vgl. Müller-Harang, Ulrike: „Carl Ruland und das Goethe-Nationalmu- 
Befürworter des Potsdamer Kopienmuseums kannten dieses Projekt. ${ }^{71}$ Es markierte neben weiteren Projekten dieser Art, nicht zuletzt in Frankreich, ${ }^{72}$ so etwas wie eine zweite Geburt des modernen Kunstbuches nach den ersten wichtigen Schritten im 18. Jahrhundert. ${ }^{73}$

Die Pionierarbeit von Prinz Albert und Carl Ruland erfüllte also das neue kunstwissenschaftliche Bedürfnis nach Gesamtübersicht und es zog weitere fotografische Sammlungsprojekte nach sich - nicht zuletzt von Ruland selbst. ${ }^{74}$ Was bei solchen Projekten allerdings verloren ging, so empfanden es jedenfalls die Väter des Potsdamer Raffaelsaals, war die authentische Präsenz des Kunstwerks, seine räumliche Erfahrbarkeit. Und genau gegen diesen Verlust trat das Potsdamer Kopienkuriosum an, erstaunlich explizit: „Der Kunst“, heißt es im Vorwort zum Verzeichnis des Raffaelsaals (1861),

[...] droht nicht geringer Nachtheil durch die industrielle Richtung unserer Zeit.

[... Die mechanischen Vervielfältigungsmittel verbreiten die Produkte der Kunst auf industrielle Weise bis in die entlegensten Winkel. Natürlich geschieht diese Extensität auf Kosten der Intensität und es stände zu befürchten, das wir zwar einen größeren Markt, aber auch nur um so leichtere Waare erhalten werden. [...] Das beste Mittel gegen die drohende Überfluthung des Seichten und MittelmäBigen, ist der Cultus des Erhabensten und Schönsten, was uns die Kunst gebracht. Der Rafaelcultus ist ein solches Mittel. [...] Die Zusammenstellung von mehr als Vierzig Hauptwerken des unsterblichen Meisters in Einem Raume, wie im Rafael-Saale, grösstentheils in ausgezeichneten Copien, bringt augenscheinlich auf empfängliche Gemüther eine Totalwirkung hervor, die geeignet erscheint, jene nachtheiligen Einflüsse zu paralysieren. ${ }^{75}$

seum“, in: Gert-Dieter Ulferts / Thomas Föhl (Hrsg.): Von der Kunstkammer zum Neuen Museum. 300 Jabre Sammlungen und Museen in Weimar. Kolloquium zu Ebren Rolf Bothe. München 2003, S. 144-161.

${ }^{71}$ Vgl. Bussler: Der Rafael-Saal, S. 64. Der Hinweis, Waagen habe über Prinz Alberts Projekt im Kunstblatt geschrieben, scheint nicht zuzutreffen.

72 Vgl. z. B. Blanc, Charles: L'oeuvre de Rembrandt reproduit par la photographie. Paris 1853-58. Für eine Übersicht über die ersten photographischen Reproduktionen von Kunstwerken in England und Frankreich und über die Debatten, die sie auslösten, vgl. McCauley, Elizabeth Anne: „Art Reproduction for the Masses“, in: Dies. (Hrsg.): Industrial Madness, Commercial Photography in Paris, 1848-1871. New Haven 1994, S. 265-300; sowie Hamber, Anthony J.: A Higher Branch of the Art. Photographing the Fine Arts in England, 1839-1880. London/Amsterdam 1996, S. 51-78.

${ }^{73}$ Vgl. Haskell, Francis: Die schwere Geburt des Kunstbuchs. Berlin 1993.

${ }^{74}$ Vgl. z. B. Ruland, Carl: Die Schätze des Goethe-National-Museums in Weimar. Sechzig photographische Aufnabmen nach den Originalen in Lichtdruck. Einleitung und erläuternde Texte vom Director C. Ruland, hrsg. von Louis Held. Weimar 1887.

${ }^{75}$ Vgl. Bussler: Der Rafael-Saal, 1861, S. 64. 
Diese wunderbare Textstelle bedarf keines langen Kommentars: Der Raffaelsaal wurde hier als scharfe Antwort auf vom Raum losgelösten, technischen Sammlungsbemühungen stilisiert. Anstelle der von der Wissenschaft geforderten bloßen „Totalübersicht“ trat hier die „Totalwirkung“ hervor, die die räumliche Dimension in der Erfahrung von Kunstwerken in den Vordergrund stellte. Anders gesagt: Wir beobachten hier (nicht ohne Rührung), wie erstaunlich früh mit museumsspezifischen Mitteln und Begriffen genau das thematisiert wurde, was Walter Benjamin - sich bezeichnenderweise auf Raffaels Sixtinische Madonna stützend - ein halbes Jahrhundert später theoretisch auf den Punkt brachte: Den Verlust der Aura durch den Verlust des Raumes.

Die Gründung des Raffaelsaals in Potsdam stellt also einen seltsamen Versuch der Rückgewinnung des Raumes und der Re-Sakralisierung mit museographischen Mitteln dar, der künstlichen Beschaffung einer Weihestätte zu emotionalen Zwecken, eines museumstechnisch gestalteten Raums für die Kunstreligion und ihre Pilger: „Wir befinden uns hier also in einem Heiligenthume, in einem ächten Kunsttempel, dessen Stiftung wir als ein epochemachendes Ereignis überhaupt und für Potsdam ganz besonders ansehen", hieß es anläßlich der Eröffnung in den Mittheilungen des Vereins für die Geschichte Potsdams. ${ }^{76}$ Die museumsgeschichtliche Verwandtschaft dieses Vorganges mit der zeitgleich geschehenen Überführung der Sixtinischen Madonna in den neueröffneten Semperbau in Dresden (1855) ist unübersehbar. In der alten Galerie hatte die Madonna hundert Jahre lang mitten unter hunderten italienischer Gemälde gehangen. In der neuen Museumskonfiguration erhielt sie einen altarähnlichen Rahmen und einen Raum für sich alleine. Raum und Rahmen sollten die verlorengegangene, ursprüngliche Funktion des Gemäldes museographisch in Erinnerung rufen und physisch erfahrbar machen. ${ }^{77}$ Über die Wirksamkeit dieser Neuinszenierung ist einiges geschrieben worden. So zum Beispiel von Leo Tolstoi, der die Dresdner Galerie kurz nach ihrer Neueröffnung besichtigt hatte und rückblickend schrieb: „Die Sixtinische Madonna ruft keinerlei Regungen in mir wach, sondern nur eine quälende Beunruhigung darüber, daß ein Gefühl von mir erwartet wird““.78

76 Bussler: Der Rafael-Saal, 1866, S. 17.

77 Vgl. Waagen, Gustav Friedrich: Einige Bemerkungen über die neue Aufstellung, Beleuchtung und Catalogisierung der königlichen Gemäldegalerie zu Dresden. Berlin 1858, S. 4-7.

78 Tolstoi, Leo: Was ist Kunst? Pläne, Anmerkungen, Notizen von L.N. Tolstoi. Zitiert nach: Alapatow, Michael W.: Die Dresdener Galerie. Alte Meister. Dresden 1966, S. 378 . 
Gefühl und Raum: Auch im Potsdamer Raffaelsaal ging es genau um dieses Begriffspaar. Und die Urheber hegten keinen Zweifel daran, einen Tempel errichtet zu haben.

Über dreißig Jahre nach der Eröffnung des Raffaelsaals in Potsdam besichtigte ein anonym gebliebener Mitarbeiter der belgischen Zeitschrift L'Art moderne das seltsame Ensemble in der Orangerie. Die Worte, die er 1891 darüber schrieb, sind geprägt vom anti-preußischen, propagandistischen Diskurs des ausgehenden 19. Jahrhunderts. Schmeichelhaft sind sie nicht:

Ein überheblicher, militaristischer Fürst ohne Geschmack, beschloß, daß auch er im wunderschönen Park von Potsdam ein Zeugnis für den architektonischen Geist seiner Zeit hinterlassen wolle. Er ließ das sog. Orangerie-Schloß errichten, eine riesige Abscheulichkeit mit mehreren Säulenreihen, ein gräzisierendes $\mathrm{Ge}$ misch. Dieser Fürst war der preußische König, Friedrich Wilhelm IV. Im großen Saal der Orangerie, einer überdimensionierten und eisigen Halle, hielt man es für angebracht, gemalte Kopien aller berühmter Raffaelgemälde zu versammeln. Fazit: es ist zum Weglaufen! Die Befürworter des Kopienmuseums könnten sich sicher sein, in Potsdam den Suizid ihres Projekts zu finden. ${ }^{79}$

Worauf der anonyme Verfasser hier anspielte, war ein langdiskutiertes Kopienmuseumsprojekt für Brüssel. Interessanterweise erwähnte er aber im selben Artikel, als lobenswertes Gegenbeispiel zur Potsdamer „Abscheulichkeit" die Einrichtung eines Museums in Leipzig, in dem die Geschichte der Kunst vom 12. Jahrhundert bis zur Gegenwart mittels Drucken und Fotografien sichtbar und erfahrbar gemacht worden war, eine „[...] sowohl technisch, als auch ideologisch lehrreiche“ Einrichtung. ${ }^{80}$ Das heutige Kunstverständnis, das von der Einmaligkeit und auratischen Unverwechselbarkeit des Kunstwerks ausgeht und dem Original eine unerhörte, ja die einzige Bedeutung beimißt, kann mit solchen Sammlungen, ob fotografisch schwarzweiß oder handwerklich bunt kaum noch etwas anfangen. Kulturhistorisch sind sie allerdings ein wertvolles Zeugnis sowohl für die räumlich museale Übersetzung kunstwissenschaftlicher Ansprüche als auch für die verschiedenen, transnationalen Mechanismen der Aneignung, die das 19. Jahrhundert hervorgebracht hat.

${ }^{79}$ N.N.: „A propos d'un musée de copies“, in: L'art moderne. Revue critique des arts et de la littérature (Brüssel), 41 (Oktober 1891), S. 327.

${ }^{80}$ Ebd. 


\section{Literaturverzeichnis}

\section{Quellen}

Berlin, Geheimes Staatsarchiv, 1. HA Rep. 89 Geh. Zivilkabinett, jüngere Periode, Akten betreffend verschiedene Maler, die u.a. als Kopisten für den Raffael-Saal tätig waren.

Berlin, Geheimes Staatsarchiv, 1. HA Rep. 89 Geh. Zivilkabinett, jüngere Periode, Nr. 19810: Gutachten von Alois Hirt vom 30. September 1821: „Acta des königl. Civil-Cabinets betr. die dem Maler Ternite bewilligten Unterstützungen, die von demselben eingereichen Gemälde und Kunstsachen und sonstige Angelegenheiten derselben 1812-1855“, fol. 79.

N.N.: „Etwas über die Pariser Gemäldeausstellung von 1810-11“, in: Paris, Wien und London, 1/1811, S. 227-231.

N.N. (Hrsg.): Verzeichniss von Kunst-und Literatur-Werken welche zum Besten Verwundeter öffentlich ausgestellt und dargelegt, zum Theil auch geschenkt und käuflich sind, Berlin 1814.

N.N. (Hrsg.): Verzeichnis von Gemälden und Kunstwerken, welche durch die Tapferkeit der vaterländischen Truppen wieder erobert worden und auf Verfügung eines Hohen Ministerii des Innern in den Sälen der königl. Akademie der Künste zu Gunsten der verwundeten Krieger des Vaterlandes [...] öffentlich ausgestellt sind. Berlin 1815.

N.N.: „A propos d'un musée de copies“, in: L'art moderne. Revue critique des arts et de la littérature (Brüssel), 41 (Oktober 1891), S. 327.

Blanc, Charles: L'oeuvre de Rembrandt reproduit par la photographie. Paris 1853-58.

Bussler, Robert: „Der Rafaelsaal im neuen Orangerie-Gebäude“, in: Louis Schneider (Hrsg.): Mittheilungen des Vereins für die Geschichte Potsdams. Bd. 2, Potsdam 1866, S. 17-22.

-: Der Rafael-Saal. Verzeichnis der im königlichen Orangeriehause zu Sans-Souci auf allerböchsten Befehl aufgestellten Copien nach Gemälden von Rafael Sanzio. Nachdr. der 2. Auflage [1861]. Bildanhang und Kommentar von Gerd Bartoschek. Potsdam 1983.

Kugler, Franz: „Einige Bedenken über Raphaels Kreuztragung, nach Maassgabe der Schlesinger'schen Kopie“, in: Deutsches Kunstblatt, 1850, Nr. 14, abgedruckt in: Kugler, Franz: Kleine Schriften und Studien zur Kunstgeschichte. 2. Teil, Stuttgart 1854, S. 594-599.

Nicolai, Friedrich: Beschreibung einer Reise durch Deutschland und die Schreiz im Jahr 1781. Berlin/Stettin 1784

Raczynski, A.[thanasius]: Geschichte der neueren deutschen Kunst. 3 Bde. Berlin 1836-41.

Ruland, Carl: Die Schätze des Goethe-National-Museums in Weimar. Sechzig photographische Aufnabmen nach den Originalen in Lichtdruck. Einleitung und erläuternde Texte vom Director C. Ruland, hrsg. von Louis Held. Weimar 1887.

Ruland, Charles (Hrsg.): The works of Raphael Santi da Urbino as represented in the Raphael collection in the Royal Library at Windsor Castle, formed by H. R. H. the Prince Consort, 185?-1861 and completed by Her Majesty Queen Victoria. Weimar 1876.

Schinkel, Karl Friedrich / Waagen, Gustav Friedrich: „Über die Aufgabe der Berliner Galerie“, August 1828, abgedruckt in: Friedrich Stock: „Urkunden zur Vorgeschichte des Berliner Museums“, in: Jb. der preußischen Kunstsammlungen 49/1928, Beiheft, S. 65-174 und 51/1930, S. 205-222.

Seume, Johann Gottfried [1801]: Spaziergang nach Syrakus im Jahre 1802. Hrsg. von Albert Meier. München 2002. 
Stock, Friedrich: „Urkunden zur Vorgeschichte des Berliner Museums“, in: Jb. der preuBischen Kunstsammlungen 49 (1928), Beiheft, S. 65-174 und 51 (1930), S. 205-222.

Uffenbach, Zacharias Conrad von: Merkwürdige Reisen durch Niedersachsen Holland und Engelland, 1709. 3 Bde. Ulm 1753/54.

Waagen, Gustav Friedrich: Einige Bemerkungen über die neue Aufstellung, Beleuchtung und Catalogisierung der königlichen Gemäldegalerie zu Dresden. Berlin 1858.

-: Verzeichniß der Copien und der Kupferstiche nach Raphael so wie einiger Originalzeichnungen dieses Meisters welche in der Rotunde des Königlichen Museum ausgestellt worden sind. Berlin 1848.

W., G.: „Lebende Bilder“, in: Journal für Kunst und Kunstsachen, Künsteleien und Mode, 12/1811, S. 289-309, hier: S. 295.

\section{Forschungsliteratur}

Alapatow, Michael W.: Die Dresdener Galerie. Alte Meister. Dresden 1966.

Asser, Saskia: „Rembrandt in photographische staat: Charles Blanc, Bisson frères en „L'Oeuvre de Rembrandt reproduit par la photographie,' 1853-1858“, in: Bulletin van bet Rijksmuseum, 48/2000, S. 170-199.

Bartoschek, Gerd / Hüneke, Saskia / Paepke, Karola: „Der Raffaelsaal: Ein Zeugnis schwärmerischer Verehrung“, Museumsjournal, Heft Nr. II/1993, S. 42-44.

-: „Ein Zeugnis schwärmerischer Verehrung. Geschichte und Restaurierung des Raffaelsaales“, in: MuseumsJournal, 7/1993, 2, S. 42-44.

Becker, Wolfgang: Paris und die deutsche Malerei. München 1971.

Bozenhard, Emanuel: Bemerkungen auf einer Reise von Kopenhagen nach Wien im Jahr 1793. Hamburg 1795.

Cuzin, Jean-Pierre / Dupuy, Marie-Anne (Hrsg.): Copier - Créer. De Turner à Picasso: 300 auvres inspirées par les maîtres du Louvre. Ausstellungskatalog, Musée du Louvre. Paris 1993.

Dahmen, Stephan: Die Bayernfenster des Kölner Doms 1844-1848: Kirchenausstattung zwischen Kunst, Theologie und Politik. Köln 2008.

Dlugaiczyk, Martina / Markschies, Alexander: Mustergültig. Gemäldekopien in neuem Licht. Das Reiff-Museum der RWTH Aachen. Katalog zur Ausstellung im Suermondt-Ludwig-Museum, Aachen 2008/09. München 2008.

Dönike, Martin (Hrsg.): Friedrich Bury, Briefe aus Italien an Goethe und Anna Amalia. Göttingen 2007.

Frank, Christoph: „L'arte e l'architettura romane nella corrispondenza di Caterina II di Russia", in: Nicola Navone (Hrsg.): Dal mito al progetto. La cultura architettonica dei maestri italiani e ticinesi nella Russia neoclassica. Mendrisio 2004, S. 60-77.

Hamber, Anthony J.: A Higher Branch of the Art. Photographing the Fine Arts in England, 1839-1880. London/Amsterdam 1996, S. 51-78.

Haskell, Fancis: The Ephemeral Museum. Old Master Paintings and the Rise of the Art Exbibition. New Haven/London 2000.

-: Die schwere Geburt des Kunstbuchs. Berlin 1993.

Jooss, Birgit: Lebende Bilder. Körperliche Nachabmung von Gruppenbildern in der Goethezeit. Berlin 1999.

Maaz, Bernhard: Christian Friedrich Tieck (1776-1851). Leben und Werk unter besonderer Berücksichtigung seines Bildnisschaffens, mit einem Werkverzeichnis. Berlin 1995. 
McCauley, Elizabeth Anne: „Art Reproduction for the Masses“, in: Dies. (Hrsg.): Industrial Madness, Commercial Photography in Paris, 1848-1871. New Haven 1994, S. $265-300$.

Müllejans-Dickmann, Rita / Haffner, Dorothee / Felbinger, Udo: Carl Joseph Begas (1794-1854). Blick in die Heimat. Heinsberg 1994.

Müller-Harang, Ulrike: „Carl Ruland und das Goethe-Nationalmuseum“, in: GertDieter Ulferts / Thomas Föhl (Hrsg.): Von der Kunstkammer zum Neuen Museum. 300 Jahre Sammlungen und Museen in Weimar. Kolloquium zu Ebren Rolf Bothe. München 2003, S. 144-161.

Nipperdey, Thomas: „Der Kölner Dom als Nationaldenkmal“, in: Ders.: Nachdenken über die deutsche Geschichte. Essays. München 1986, S. 189-207.

Osterkamp, Ernst: „Maria, Auguste und die Madonna. Die Bedeutung Raffaels für Goethe, erläutert am Beispiel von zwei Gedichten an Personen.", in: Gerhard Neuman / David E. Wellbery (Hrsg.): Die Gabe des Gedichts. Goethes Lyrik im Wechsel der Töne. Freiburg 2008, S. 185-207.

-: „Raffael-Forschung von Fiorillo bis Passavant", in: Studi germanici, nuova serie, XXXVIII/2000, 3, S. 403-426.

Putscher, Marielene: Raphaels Sixtinische Madonna: das Werk und seine Wirkung. Tübingen 1955.

Roberts, Helene E. (Hrsg.): Art History through the Camera's Lens. London/Amsterdam 1995.

Roenne, Bettina von: Ein Architekt rabmt Bilder. Karl Friedrich Schinkel und die Berliner Gemäldegalerie. Berlin 2007.

Ruhmer, Eberhard (Hrsg.): Schack-Galerie. Vollständiger Katalog. 2 Bde. München 1969.

Savoy, Bénédicte (Hrsg.): Tempel der Kunst. Die Entstehung des öffentlichen Museums in Deutschland. 1701-1815. Mainz 2006.

-: Patrimoine annexé. 2 Bde. Paris 2003.

Scherkl, Robert: „Charles Blancs Musee des copies. Kopien wie Originale?“, in: Zeitschrift für Kunstgeschichte, 63/2000, 3, S. 358-371.

Schröter, Elisabeth: „Raffael-Kult und Raffael-Forschung. Johann David Passavant und seine Raffael-Monographie im Kontext der Kunst und Kunstgeschichte seiner Zeit“, in: Römisches Jabrbuch, 26/1990, S. 303-393.

Stockhausen, Tillmann von: Gemäldegalerie Berlin. Die Geschichte ibrer Erwerbungspolitik. Berlin 2000.

Strittmatter, Anette: Das, Gemäldekopieren 'in der deutschen Malerei zzeischen 1780 und 1860. Münster 1998.

Тютрина, Елена: Парадные заль академии художеств, Российская академия художеств - научко-исследовательский музей. Sankt Petersburg 2004 [Tjutrina, Elena: Paradnyje zaly akademii xudožestv, Rossijjkaja akademija xudožestv - naučnoissledovatel'skij muzej. Sankt Petersburg 2004].

Wagner, Cornelia: Arbeitsweisen und Anschaunngen in der Gemälderestaurierung um 1800 (= Veröffentlichungen des Instituts für Kunsttechnik und Konservierung im Germanischen Nationalmuseum, Bd. 2). München 1988.

Windholz, Angela: „Raffael als alter ego der Preußischen Monarchie. Zur bildpolitischen Intention des Raffaelsaals in Sanssouci“, in: Marburger Jabrbuch für Kunstwissenschaft, Bd. 35 (2008), S. 215-265. 